\title{
A compositional Semantics for CHR
}

\author{
Giorgio Delzanno \\ Dip. di Informatica e Scienze \\ dell'Informazione \\ Università di Genova \\ via Dodecaneso 35, 16146 \\ Genova, Italy \\ giorgio@disi.unige.it
}

\author{
Maurizio Gabbrielli \\ Dipartimento di Scienze \\ dell'Informazione \\ Università di Bologna \\ Mura A.Zamboni 7, 40127 \\ Bologna, Italy \\ gabbri@cs.unibo.it
}

\author{
Maria Chiara Meo \\ Dipartimento di Scienze \\ Università di Chieti \\ Viale Pindaro 42, 65127 \\ Pescara, Italy \\ cmeo@unich.it
}

\begin{abstract}
Constraint Handling Rules (CHR) are a committed-choice declarative language which has been designed for writing constraint solvers. A CHR program consists of multi-headed guarded rules which allow one to rewrite constraints into simpler ones until a solved form is reached.

CHR has received a considerable attention, both from the practical and from the theoretical side. Nevertheless, due the use of multiheaded clauses, there are several aspects of the CHR semantics which have not been clarified yet. In particular, no compositional semantics for CHR has been defined so far.

In this paper we introduce a fix-point semantics which characterizes the input/output behavior of a CHR program and which is andcompositional, that is, which allows to retrieve the semantics of a conjunctive query from the semantics of its components. Such a semantics can be used as a basis to define incremental and modular analysis and verification tools.
\end{abstract}

\section{INTRODUCTION}

Constraint Handling Rules (CHR) [11, 12] are a committed-choice declarative language which has been specifically designed for writing constraint solvers. The first constraint logic languages used mainly built-in constraint solvers designed by following a "black box" approach. This made hard to modify, debug, and analyze a specific solver. Moreover, it was very difficult to adapt an existing solver to the needs of some specific applications, and this was soon recognized as a serious limitation since often practical applications involve application specific constraints.

By using CHR one can easily introduce specific user-defined constraints and the related solver into an host language. In fact, a CHR program consists of (a set of) multi-headed guarded simplification and propagation rules which are specifically designed to implement the two most important operations involved in the constraint solv- ing process: Simplification rules allow to replace constraints by simpler ones, while preserving their meaning. Propagation rules are used to add new redundant constraints which do not modify the meaning of the given constraint and which can be useful for further reductions. It is worth noting that the presence of multiple heads in CHR is an essential feature which is needed in order to define reasonably expressive constraint solvers (see the discussion in [12]). However, such a feature, which differentiates this proposal from many existing committed choice logic languages, complicates considerably the semantics of CHR, in particular it makes very difficult to obtain a compositional semantics, as we argue below. This is unfortunate, as compositionality is an highly desirable property for a semantics. In fact, a compositional semantics provides the basis to define incremental and modular tools for software analysis and verification, and these features are essential in order to deal with partially defined components. Moreover, in some cases, modularity allows to reduce the complexity of verification of large systems by considering separately smaller components.

In this paper we introduce a fix-point semantics for CHR which characterizes the input/output behavior of a program and which is and-compositional, that is, which allows to retrieve the semantics of a conjunctive query from the semantics of its components.

In general, due to the presence of synchronization mechanisms, the input/ouput semantics is not compositional for committed choice logic languages and for most concurrent languages in general. Indeed, the need for more complicate semantic structures based on traces was recognized very early as a necessary condition to obtain a compositional model, first for dataflow languages [13] and then in the case of many other paradigms, including imperative concurrent languages [8] and concurrent constraint and logic languages [6].

When considering CHR this basic problem is further complicated: due to the presence of multiple heads the traces consisting of sequences of input/ouput pairs, analogous to those used in the above mentioned works, are not sufficient to obtain a compositional semantics. Intuitively the problem can be stated as follows. A CHR rule $r: A, B \Leftrightarrow c \mid C$ cannot be used to rewrite a goal $A$, no matter how the variables are constrained (that is, for any input constraint), because the goal consists of a single atom $A$ while the head of the rule contains two atoms $A, B$. Therefore, if we considered a semantics based on input/ouput traces, we would obtain the empty denotation for the goal $A$ in the program consisting of the rule $r$ plus some rules defining $C$. Analogously for the goal $B$. On the other hand, the rule $r$ can be used to rewrite the goal $A, B$. Therefore, provided that the semantics of $C$ is not empty, the semantics 
of $A, B$ is not empty and cannot be derived from the semantics of $A$ and $B$, that is, such a semantics is not compositional. It is worth noting that even restricting to a more simple notion of observable, such as the results of terminating computations, does not simplify this problem. In fact, differently from the case of ccp languages, also the semantic based on these observables (usually called resting points) is not compositional for $\mathrm{CHR}$.

Our solution to obtain a compositional model is to use an augmented semantics based on traces which includes at each steps two "assumptions" on the external environment and two "outputs" of the current process: Similarly to the case of the models for ccp, the first assumption is made on the constraints appearing in the guards of the rules, in order to ensure that these are satisfied and the computation can proceed. The second assumption is specific to our approach and contains atoms which can appear in the heads of rules. This allows us to rewrite a goal $G$ by using a rule whose head $H$ properly contains $G$ : While this is not possible with the standard CHR semantics, we allow that by assuming that the external environment provides the "difference" $H$ minus $G$ and by memorizing such an assumption. The first output element is the constraint produced by the process, as usual. We also memorize at each step also a second output element, consisting of those atoms which are not rewritten in the current derivation and which could be used to satisfy some assumptions (of the second type) when composing sequences representing different computations. Thus our model is based on sequences of quadruples, rather than of simple input/output pairs.

Our compositional semantics is obtained by a fixpoint construction which uses an enhanced transitions system implementing the rules for assumptions described above. We prove the correctness of the semantics w.r.t. a notion of observables which characterizes the input/ouput behavior of terminating computations where the original goal has been completely reduced to built-in constraints. We will discuss later the extensions needed in order to characterize different notions of results, such as the "qualified answers" used in [12].

The remaining of this paper is organized as follows. Next section introduces some preliminaries about CHR and its operational semantics. Section 3 contains the definition of the compositional semantics, while section 4 presents the compositionality and correctness results. Section 6 concludes by discussing directions for future work.

Note for the reviewer: To avoid the reference to a technical report, for the convenience of the reviewer we include also a technical appendix containing the proofs of some lemmas used in the paper. This Appendix however is not meant to be part of the submitted paper.

\section{PRELIMINARIES}

In this section we first introduce some preliminary notions and then define the CHR syntax and operational semantics. Even though we try to provide a self-contained exposition, some familiarity with constraint logic languages and first order logic could be useful.

We first need to distinguish the constraints handled by an existing solver, called built-in (or predefined) constraints, from those defined by the CHR program, user-defined (or CHR) constraints. An atomic constraint is a first-order predicate (atomic formula). By assuming to use two disjoint sorts of predicate symbols we then distinguish built-in atomic constraints from CHR atomic constraints.
A built-in constraint $c$ is defined by

$$
c::=a|c \wedge c| \exists_{x} a
$$

where $a$ is an atomic built-in constraint ${ }^{1}$. For built-in constraints we assume given a theory $\mathrm{CT}$ which describes

On the other hand, according to the usual CHR syntax, we assume that a user-defined constraint is a conjunction of atomic userdefined constraints. We use $c, d$ to denote built-in constraints, $g, h, k$ to denote CHR constraints and $a, b$ to denote both built-in and userdefined constraints (we will call these generically constraints). The capital versions of these notations will be used to denote multisets of constraints. Furthermore we denote by $\mathcal{U}$ the set of user-defined constraints and by $\mathcal{B}$ the set of built-in constraints.

We will often use "," rather than $\wedge$ to denote conjunction and we will often consider a conjunction of atomic constraints as a multiset of atomic constraints. In particular, we will use this notation based on multisets in the syntax of CHR. The notation $\exists_{-V} \phi$ where $V$ is a set of variables denotes the existential closure of a formula $\phi$ with the exception of the variables $V$ which remain unquantified. $F v(\phi)$ denotes the free variables appearing in $\phi$ and we denote by - the concatenation of sequences and by $\varepsilon$ the empty sequence. Furthermore $\uplus$ denotes the multi-set union, while we consider $\backslash$ as an overloaded operator used both for set and multi-set difference (the meaning depends on the type of the arguments).

We are now ready to define the CHR syntax.

Definition 1. (Syntax) [12] A CHR simplification rule has the form $H \Leftrightarrow c \mid B$ while a CHR propagation rule has the form $H \Rightarrow c \mid B$ where $H$ is a non-empty multiset of user-defined constraints, $c$ is a built-in constraint and $B$ is a possibly empty multi-set of constraints.

A CHR program is a set of CHR simplification and propagation rules. A CHR goal is a multiset of (both user-defined an built-in) constraints.

We prefer to use multisets rather than sequences (as in the original CHR papers) since multisets appear to correspond more precisely to the nature of CHR rules. We denote by Goals the set of all goals.

We describe now the operational semantics of CHR as provided by [12] by using a transition system $T_{s}=\left(\operatorname{Conf}_{s}, \longrightarrow_{s}\right)\left(_{s}\right.$ here stands for "standard", as opposed to the semantics we will use later). Configurations in $\operatorname{Conf}_{s}$ are triples of the form $\langle G, K, d\rangle$ where $G$ are the constraints that remain to be solved, $K$ are the user-defined constraints that have been accumulated and $d$ are the built-in constraints that have been simplified ${ }^{2}$.

\footnotetext{
${ }^{1}$ We could consider more generally first order formulas as built-in constraints, as far as the results presented here are concerned.

${ }^{2}$ In [12] triples of the form $\langle G, K, d\rangle_{\mathcal{V}}$ were used, where the annotation $\mathcal{V}$, which is not changed by the transition rules, is used to distinguish the variables appearing in the initial goal from the variables which are introduced by the rules. We can avoid such an indexing by explicitly referring to the original goal.
} 
An initial configuration has the form

$$
\langle G, \emptyset, \emptyset\rangle
$$

and consists of a goal $G$, an empty user-defined constraint and an empty built-in constraint.

\section{A final configuration has either the form}

$$
\langle G, K,\{\text { false }\}\rangle,
$$

when it is failed, i.e. when it contains an inconsistent built-in constraint store represented by the unsatisfiable constraint false, or has the form

$$
\langle G, K, d\rangle
$$

when it is successfully terminated since there are no applicable rules.

Given a program $P$, the transition relation $\longrightarrow s \subseteq$ Conf $\times$ Conf is the least relation satisfying the rules in Table 1 (for the sake of simplicity, we omit indexing the relation with the name of the program). The Solve transition allows to update the constraint store by taking into account a built-in constraint contained in the goal. Without loss of generality, we will assume that $F v\left(d^{\prime}\right) \subseteq$ $F v(c) \cup F v(d)$. The Introduce transition is used to move a userdefined constraint from the goal to the CHR constraint store, where it can be handled by applying CHR rules. The transitions Simplify and Propagate allow to rewrite user-defined constraints (which are in the CHR constraint store) by using rules from the program. As usual, in order to avoid variable names clashes, both these transitions assume that clauses from the program are renamed apart, that is assume that all variables appearing in a program clause are fresh ones. Both the Simplify and Propagate transitions are applicable when the current store $(d)$ is strong enough to entail the guard of the rule $(c)$, once the parameter passing has been performed (this is expressed by the equation $H=H^{\prime}$ ). Note that, due to the existential quantification over the variables $x$ appearing in $H$, in such a parameter passing the information flow is from the actual parameter (in $H^{\prime}$ ) to the formal parameters $(H)$, that is, it is required that the constraints $H^{\prime}$ which have to be rewritten are an instance of the head $H$. When applied, both these transitions add the body $B$ of the rule to the current goal and the equation $H=H^{\prime}$, expressing the parameter passing mechanism, to the built-in constraint store. The difference between Simplify and Propagate is in the fact that while the former transition removes the constraints $H^{\prime}$ which have been rewritten from the CHR constraint store, this is not the case for the latter.

Given a goal $G$, the operational semantics that we consider observes the final stores of computations terminating with an empty goal and an empty user-defined constraint. We call these observables success answers slightly deviating from the terminology of [12] (a goal which has a success answer is called a data-sufficient goal in [12]).

Definition 2. (Success answers) Let $P$ be a program and let $G$ be a goal. The set $\mathcal{S A}_{P}(G)$ of success answers for the query $G$ in the program $P$ is defined as follows

$$
\mathcal{S}_{P}(G)=\left\{\left\langle\exists_{-F v(G)} d\right\rangle \mid\langle G, \emptyset, \emptyset\rangle \longrightarrow_{s}^{*}\langle\emptyset, \emptyset, d\rangle \longleftrightarrow_{P}\right\} .
$$

In [12] it is considered also the following different notion of answer, obtained by computations terminating with a user-defined constraint which does not need to be empty.
Definition 3. (Qualified answers) Let $P$ be a program and let $G$ be a goal. The set $\mathcal{Q} \mathcal{A}_{P}(G)$ of qualified answers for the query $G$ in the program $P$ is defined as follows

$$
\mathcal{Q A}_{P}(G)=\left\{\left\langle\exists_{-F v(G)} K \wedge d\right\rangle \mid\langle G, \emptyset, \emptyset\rangle \longrightarrow_{s}^{*}\langle\emptyset, K, d\rangle \longrightarrow_{P}\right\} .
$$

We discuss in Section 6 the extensions needed to characterize also qualified answers. Note that both previous notions of observables characterize an input/output behavior, since the input constraint is implicitly considered in the goal.

In the remaining of this paper we will consider only simplification rules since propagation rules can be mimicked by simplification rules, as far as the results contained in this paper are concerned.

Note that in presence of propagation rules the "naive" operational semantics that we consider in this paper introduces redundant infinite computations: Since propagation rules do not remove user defined constraints (see rule Propagate in Table 1), when a propagate rule is applied it introduce an infinite computation (obtained by subsequent applications of the same rule). Note however that this does not imply that in presence of an active propagation rule the semantics that we consider are empty. In fact, the application of a simplification rule after a propagation rule can cause the termination of the computation, by removing the atoms which are needed by the head of the propagation rule. It is also possible to define a more refined operational semantics (see [1]) which avoid these infinite computations by allowing to apply at most once a propagation rule to the same constraints. We discuss in Section 5 the modifications needed in our construction to take into account this more refined semantics.

\section{A COMPOSITIONAL TRACE SEMAN- TICS}

Given a program $P$, we say that a semantics $\mathcal{S}_{P}$ is and-compositional if $\mathcal{S}_{P}(A, B)=\mathcal{C}\left(\mathcal{S}_{P}(A), \mathcal{S}_{P}(B)\right)$ for a suitable composition operator $\mathcal{C}$ which does not depend on the program $P$. As mentioned in the introduction, due to the presence of multiple heads in $\mathrm{CHR}$, the semantics which associate to a program $P$ the function $\mathcal{S} \mathcal{A}_{P}$ is not and-compositional, since goals which have the same input/ouput behavior can behave differently when composed with other goals. Consider for example the program $P$ consisting of the single rule

$$
g, h \Leftrightarrow \text { true } \mid c .
$$

(where $c$ is a built-in constraint). According to Definition 3 we have that $\mathcal{S} \mathcal{A}_{P}(g)=\mathcal{S} \mathcal{A}_{P}(k)=\emptyset$, while

$$
\mathcal{S A}_{P}(g, h)=\left\{\left\langle\exists_{-F v(g, h)} c\right\rangle\right\} \neq \emptyset=\mathcal{S A}_{P}(k, h) .
$$

An analogous example can be made to show that also the semantics $\mathcal{Q} \mathcal{A}$ is not and-compositional.

The problem exemplified above is different from the classic problem of concurrent languages where the interaction of non-determinism and synchronization makes the input/output observables noncompositional. For this reason, considering simply sequences of (input-output) built-in constraints is not sufficient to obtain a compositional semantics for CHR. We have to use some additional information which allow us to describe the behavior of goals in any possible and-composition without, of course, considering explicitly all the possible and-compositions. 


\begin{tabular}{|ll|}
\hline Solve & $\frac{C T \models c \wedge d \leftrightarrow d^{\prime} \text { and c is a built-in constraint }}{\langle(c, G), K, d\rangle \mathrm{s}_{s}\left\langle G, K, d^{\prime}\right\rangle}$ \\
Introduce & $\frac{\mathrm{h} \text { is a user-defined constraint }}{\langle(h, G), K, d\rangle \longrightarrow_{s}\langle G,(h, K), d\rangle}$ \\
Simplify & $\frac{H \Leftrightarrow c \mid B \in P \quad x=\operatorname{Var}(H) \quad C T=d \rightarrow \exists_{x}\left(\left(H=H^{\prime}\right) \wedge c\right)}{\left\langle G, H^{\prime} \wedge K, d\right\rangle \mathrm{s}_{s}\left\langle B \wedge G, K, H=H^{\prime} \wedge d\right\rangle}$ \\
Propagate & $\frac{H \Rightarrow c \mid B \in P \quad x=\operatorname{Var}(H) \quad C T=d \rightarrow \exists_{x}\left(\left(H=H^{\prime} \wedge c\right)\right)}{\left\langle G, H^{\prime} \wedge K, d\right\rangle \longrightarrow{ }_{s}\left\langle B \wedge G, H^{\prime} \wedge K, H=H^{\prime} \wedge d\right\rangle}$ \\
&
\end{tabular}

Table 1: The standard transition system for CHR

The basic idea of our approach is to collect in the semantics also the "missing" parts of heads which are needed in order to proceed with the computation. For example, when considering the program $P$ above, we should be able to state that the goal $g$ produces the constraint $c$, provided that the external environment (i.e. a conjunctive goal) contains the user-defined constraint $h$. In other words, $h$ is an assumption which is made in the semantics describing the computation of $g$. When composing (by using a suitable notion of composition) such a semantics with that one of a goal which contains $h$ we can verify that the "assumption" $h$ is satisfied and therefore obtain the correct semantics for $g, h$. In order to model correctly the interaction of different processes we have to use sequences, analogously to what happens with other concurrent paradigms.

This idea is developed by defining a new transition system which implements this mechanism based on assumptions for dealing with the missing parts of heads. The new transition system allows one to generate the sequences appearing in the compositional model by using a standard fix-point construction. As a first step in our construction we modify the notion of configuration used before: Since we do not need to distinguish user-defined constraints which appear in the goal from the user-defined constraints which have been already considered for reduction, we merge the first and the second components of previous triples. Thus we do not need anymore Introduce rules. On the other hand, we need the information on the new assumptions, which is added as a label of the transitions.

Thus we define a transition system $T=($ Conf,$\longrightarrow P)$ where configurations in Conf are pairs: the first component is a multi-set of indexed atoms (the goal) and the second one is a built-in constraint (the store). Indexes are associated to atoms in order to denote the point in the derivation where they have been introduced. More precisely, atoms in the original goals are labeled by 0 , while atoms introduced at the $i-t h$ derivation step are labeled by $i$. Given a program $P$, the transition relation $\longrightarrow P \subseteq$ Conf $\times$ Conf $\times \wp(\mathcal{U})$ is the least relation satisfying the rules in Table 2 (where $\wp(A)$ denotes the set consisting of all the subsets of $A$ (. Note that we consider only Solve and Simplify rules, as the other rules as previously mentioned are redundant in this context. Solve' is the same rule as before, while the Simplify' rule is modified to consider assumptions: When reducing a goal $G$ by using a rule having head $H$, the set of assumptions $K=H \backslash G$ (with $H \neq K$ ) is used to label the transition ( $\backslash$ here denotes multiset difference). Indexes allow us to distinguish identical occurrences of atoms which have been introduced in different derivation steps. We will use the no- tation $G^{\max =i}$ to indicate that $i$ is the maximal label occurring in the (non-atomic) goal $G$ and $G^{i}$ to indicate that all the atoms in $G$ are labeled by $i$. When indexes are not needed we will simply omit them. As before, we assume that program rules to be used in the new simplify rule use fresh variables to avoid names captures.

The semantics domain of our compositional semantics is based on sequences which represent derivations obtained by the transition system in Table 2. More precisely, we first consider "concrete" sequences consisting of tuples of the form $\left\langle G, c, K, G^{\prime}, d\right\rangle$ : Such a tuple represents exactly a derivation step $\langle G, c\rangle \longrightarrow^{K}\left\langle G^{\prime}, d\right\rangle$. The sequences we consider are terminated by tuples of the form $\langle G, c, \emptyset, G, c\rangle$, which represent a terminating step (see the precise definition below). Since a sequence represents a derivation, we assume that the "output" goal $G^{\prime}$ at step $i$ is equal to the "input" goal $G$ at step $i+1$, that is, we assume that if

$$
\ldots\left\langle G_{i}, c_{i}, K_{i}, G_{i}^{\prime}, d_{i}\right\rangle\left\langle G_{i+1}, c_{i+1}, K_{i+1}, G_{i+1}^{\prime}, d_{i+1}\right\rangle \ldots
$$

appears in a sequence, then $G_{i}^{\prime}=G_{i+1}$ holds.

On the other hand, the input store $c_{i+1}$ can be different from the output store $d_{i}$ produced at previous step, since we need to perform all the possible assumptions on the constraint $c_{i+1}$ produced by the external environment in order to obtain a compositional semantics. However, we assume that if

$$
\ldots\left\langle G_{i}, c_{i}, K_{i}, G_{i}^{\prime}, d_{i}\right\rangle\left\langle G_{i+1}, c_{i+1}, K_{i+1}, G_{i+1}^{\prime}, d_{i+1}\right\rangle \ldots
$$

appears in a sequence then $C T \models c_{i+1} \rightarrow d_{i}$ holds: This means that the assumption made on the external environment cannot be weaker than the constraint store produced at the previous step. This reflects the monotonic nature of computations, where information can be added to the constraint store and cannot be deleted from it. Finally note that assumptions on user-defined constraints (label $K$ ) are made only for the atoms which are needed to "complete" the current goal in order to apply a clause. In other words, no assumption can be made in order to apply clauses whose heads do not share any predicate with the current goal.

The set of the above described "concrete" sequences, which represent derivation steps performed by using the new transition system, is denoted by $\mathcal{S} e q$.

¿From these concrete sequences we extract some more abstract sequences which are the objects of our semantic domain: From each tuple $\left\langle G, c, K, G^{\prime}, d\right\rangle$ in a sequence $\delta \in \mathcal{S} e q$ we extract a 


$$
\begin{aligned}
& \text { Solve' } \quad \frac{C T \models c \wedge d \leftrightarrow d^{\prime}}{\left\langle c \wedge G^{\text {max }=i}, d\right\rangle \longrightarrow^{\emptyset}\left\langle G^{\text {max }=i}, d^{\prime}\right\rangle} \\
& \text { Simplify, } \quad \frac{\mathrm{cl}=H \leftrightarrow c \mid B \in P \quad x=F v(H) \quad G^{\text {max }=i} \neq \emptyset \quad C T \models d \rightarrow \exists_{x}\left(\left(H=\left(G^{\text {max }=i}, K\right)\right) \wedge c\right)}{\left\langle G^{\text {max }=i} \wedge A, d\right\rangle \longrightarrow^{K}\left\langle B^{i+1} \wedge A, d \wedge(H=(G, K))\right\rangle}
\end{aligned}
$$

Table 2: The transition system for the compositional semantics

tuple of the form $\langle c, K, H, d\rangle$ where we consider as before the input and output store ( $c$ and $d$, respectively) and the assumptions $(K)$, while we do not consider anymore the output goal $G^{\prime}$. Furthermore, we restrict the input goal $G$ to that part $H$ consisting of all those user-defined constraints which will not be rewritten in the (derivation represented by the) sequence $\delta$. Intuitively $H$ contains those atoms which are available for satisfying assumptions of other goals, when composing two different sequences (representing two derivations of different goals). We also assume that if $\left\langle c_{i}, K_{i}, H_{i}, d_{i}\right\rangle\left\langle c_{i+1}, K_{i+1}, H_{i+1}, d_{i+1}\right\rangle$ is in a sequence then $H_{i} \subseteq H_{i+1}$ holds, since these atoms which will not be rewritten in the derivation can only augment. We then define formally the semantic domain as follows.

Definition 4. (Sequences) The semantic domain $\mathcal{D}$ containing all the possible sequences is defined as the set

$$
\begin{gathered}
\mathcal{D}=\left\{\left\langle c_{1}, K_{1}, H_{1}, d_{1}\right\rangle \ldots\left\langle c_{n}, \emptyset, H_{n}, c_{n}\right\rangle \mid\right. \\
\quad \text { or each } j, 1 \leq j \leq n \text { and for each } i, 1 \leq i \leq n-1, \\
\\
H_{j} \text { and } K_{i} \text { are multisets of CHR indexed constraints, } \\
\\
c_{j}, d_{i} \text { are built-in constraints and } C T \models d_{i} \rightarrow c_{i}, \\
\\
\left.H_{i} \subseteq H_{i+1} \text { and } C T \models c_{i+1} \rightarrow d_{i} \text { holds }\right\} .
\end{gathered}
$$

In order to define our semantics we need two more notions. First, we define an abstraction operator $\alpha$ which extracts from the concrete sequences in $\mathcal{S} e q$ (representing exactly derivation steps) the sequences used in our semantic domain.

Definition 5. Let $\delta=\left\langle G_{1}, c_{1}, K_{1}, G_{2}, d_{1}\right\rangle \ldots\left\langle G_{n}, c_{n}, \emptyset, G_{n}, c_{n}\right\rangle$ be a sequence of derivation steps where we assume that atoms are indexed as previously specified. We say that an indexed atom $A^{j}$ is stable in $\delta$ if $A^{j}$ appears in $G_{i}$ and in $G_{i+1}$, for each $1 \leq i \leq n-1$. The abstraction operator $\alpha: \mathcal{S e q} \rightarrow \mathcal{D}$ is then defined inductively as

$$
\begin{aligned}
& \alpha(\varepsilon)=\varepsilon \\
& \alpha\left(\left\langle G, c, K, G^{\prime}, d\right\rangle \cdot \delta^{\prime}\right)=\langle c, K, H, d\rangle \cdot \alpha\left(\delta^{\prime}\right)
\end{aligned}
$$

where $H$ is the multiset consisting of all the indexed atoms in $G$ which are stable in $\left\langle G, c, K, G^{\prime}, d\right\rangle \cdot \delta^{\prime}$.

Then we need the notion of "compatibility" of a tuple w.r.t. a sequence. To this aim we first provide some further notation: Given a sequence of derivation steps

$\delta=\left\langle G_{1}, c_{1}, K_{1}, G_{2}, d_{1}\right\rangle\left\langle G_{2}, c_{2}, K_{2}, G_{3}, d_{2}\right\rangle \ldots\left\langle G_{n}, c_{n}, \emptyset, G_{n}, c_{n}\right\rangle$

we denote by length $(\delta)$ and by instore $(\delta)$ the length of the derivation $\delta$ and the first input store $c_{1}$, respectively. Moreover using $t$ as a shorthand for the tuple $\left\langle G_{1}, c_{1}, K_{1}, G_{2}, d_{1}\right\rangle$ we define

$$
\begin{aligned}
& V_{l o c}(t)=F v\left(G_{2}, d_{1}\right) \backslash F v\left(G_{1}, c_{1}, K_{1}\right), \\
& V_{\text {ing }}(\delta)=F v\left(G_{1}\right), \\
& V_{a s s}(\delta)=\bigcup_{i=1}^{n-1} F v\left(K_{i}\right), \\
& V_{\text {stable }}(\delta)=F v\left(G_{n}\right), \\
& V_{\text {constr }}(\delta)=\bigcup_{i=1}^{n-1} F v\left(d_{i}\right) \backslash F v\left(c_{i}\right) \text { and } \\
& V_{l o c}(\delta)=\bigcup_{i=1}^{n-1} F v\left(G_{i+1}, d_{i}\right) \backslash F v\left(G_{i}, c_{i}, K_{i}\right) .
\end{aligned}
$$

We then define a compatibility as follows.

Definition 6. Let $t=\left\langle G_{1}, c_{1}, K_{1}, G_{2}, d_{1}\right\rangle$ a tuple representing a derivation step for the goal $G_{1}$ and let

$$
\delta=\left\langle G_{2}, c_{2}, K_{2}, G_{3}, d_{2}\right\rangle \ldots\left\langle G_{n}, c_{n}, \emptyset, G_{n}, c_{n}\right\rangle
$$

be a sequence of derivation steps for $G_{2}$. We say that $t$ is compatible with $\delta$ if the following hold:

1. $C T \models$ instore $(\delta) \rightarrow d_{1}$,

2. $V_{l o c}(\delta) \cap F v(t)=\emptyset$,

3. for $i \in[2, n], V_{l o c}(t) \cap F v\left(c_{i}\right) \subseteq \bigcup_{j=1}^{i-1} F v\left(d_{j}\right) \cup V_{\text {stable }}(\delta)$ and

4. $V_{l o c}(t) \cap V_{a s s}(\delta)=\emptyset$.

Note that if $t$ is compatible with $\delta$ then, by using the notation above, $t \cdot \delta$ is a sequence of derivation steps for $G_{1}$. We can now define the compositional semantics.

Definition 7. (Compositional semantics) Let $P$ be a program and let $G$ be a goal. The compositional semantics of $G$ in the program $P, \mathcal{S}_{P}:$ Goals $\rightarrow \wp(\mathcal{D})$, is defined as

$$
\mathcal{S}_{P}(G)=\alpha\left(\mathcal{S}_{P}^{\prime}(G)\right)
$$

where $\alpha$ is the pointwise extension to sets of the operator given in Definition 5 and $\mathcal{S}_{P}^{\prime}:$ Goals $\rightarrow \wp(\mathcal{S e q})$ is defined as follows:

$$
\begin{aligned}
\mathcal{S}^{\prime}{ }_{P}(G)= & \left\{\left\langle G, c, K, G^{\prime}, d\right\rangle \cdot \delta \in \mathcal{S} e q \mid\right. \\
& C T \not c \leftrightarrow \text { false, }\langle G, c\rangle \longrightarrow^{K}\left\langle G^{\prime}, d\right\rangle \\
& \text { and } \delta \in \mathcal{S}_{P}\left(G^{\prime}\right) \text { for some } \delta \text { such that } \\
& \left.\left\langle G, c, K, G^{\prime}, d\right\rangle \text { is compatible with } \delta\right\} \\
\cup & \\
& \{\langle G, c, \emptyset, G, c\rangle \in \mathcal{S} e q\} .
\end{aligned}
$$


Formally $\mathcal{S}_{P}^{\prime}(G)$ is defined as the least fixed-point of the corresponding operator $\Phi \in($ Goals $\rightarrow \wp(\mathcal{S} e q)) \rightarrow$ Goals $\rightarrow \wp(\mathcal{S} e q)$ defined by

$$
\begin{aligned}
\Phi(I)(G)= & \left\{\left\langle G, c, K, G^{\prime}, d\right\rangle \cdot \delta \in \mathcal{S} e q \mid\right. \\
& C T \not \neq c \leftrightarrow \text { false, }\langle G, c\rangle \longrightarrow^{K}\left\langle G^{\prime}, d\right\rangle \\
& \text { and } \delta \in I\left(G^{\prime}\right) \text { for some } \delta \text { such that } \\
& \left.\left\langle G, c, K, G^{\prime}, d\right\rangle \text { is compatible with } \delta\right\} \\
\cup & \\
& \{\langle G, c, \emptyset, G, c\rangle \in \mathcal{S} e q\} .
\end{aligned}
$$

In the above definition, $I:$ Goals $\rightarrow \wp(\mathcal{S} e q)$ stands for a generic interpretation assigning to a goal a set of sequences, and the ordering on the set of interpretations Goals $\rightarrow \wp(\mathcal{S e q})$ is that of (pointwise extended) set-inclusion. It is straightforward to check that $\Phi$ is continuous (on a CPO), thus standard results ensure that the fixpoint can be calculated by $\sqcup_{n \geq 0} \phi^{n}(\perp)$, where $\phi^{0}$ is the identity map and for $n>0, \phi^{n}=\phi \circ \phi^{n-1}$ (see for example [9]).

\section{COMPOSITIONALITY AND CORRECT- NESS}

In this section we prove that the semantics defined above is andcompositional and correct w.r.t. the observables $\mathcal{S A}_{P}$.

In order to prove the compositionality result we first need to define how two sequences describing a computation of $A$ and $B$, respectively, can be composed in order to obtain a computation of $A, B$. Such a composition is defined by the (semantic) operator $\|$ which performs an interleaving of the actions described by the two sequences and then eliminates the assumptions which are satisfied in the resulting sequence. For technical reasons, rather than modifying the existing sequences, the elimination of satisfied assumptions is performed on new sequences which are generated by a closure operator $\eta$ defined as follows.

Definition 8. Let $W$ be a multiset of indexed atoms and let $\sigma$ be a sequence in $\mathcal{D}$ of the form

$$
\left\langle c_{1}, K_{1}, H_{1}, d_{1}\right\rangle \ldots\left\langle c_{n}, K_{n}, H_{n}, d_{n}\right\rangle .
$$

We denote by $\sigma \backslash W$ the sequence

$$
\left\langle c_{1}, K_{1}, H_{1} \backslash W, d_{1}\right\rangle \ldots\left\langle c_{n}, K_{n}, H_{n} \backslash W, d_{n}\right\rangle
$$

where the multisets difference $H_{i} \backslash W$ considers indexes.

The operator $\eta: \wp(\mathcal{D}) \rightarrow \wp(\mathcal{D})$ is defined as follows. Given $S \in \wp(\mathcal{D}), \eta(S)$ is the least set satisfying the following conditions:

$$
\begin{aligned}
& \text { 1. } S \subseteq \eta(S) \text {; } \\
& \text { 2. if } \sigma^{\prime} \cdot\langle c, K, H, d\rangle \cdot \sigma^{\prime \prime} \in S \text { then }\left(\sigma^{\prime} \cdot\left\langle c, K \backslash K^{\prime}, H, d\right\rangle .\right. \\
& \left.\sigma^{\prime \prime}\right) \backslash W \in \eta(S)
\end{aligned}
$$

where $K^{\prime}=\left\{A_{1}, \ldots, A_{n}\right\} \subseteq K$ is a multiset such that there exists a multiset (of indexed atoms) $W=\left\{B_{1}^{j_{1}}, \ldots, B_{n}^{j_{n}}\right\} \subseteq H$ such that $C T \models c \wedge B_{i} \leftrightarrow c \wedge A_{i}$, for each $i \in[1, n]$.

A few explanations are in order. The operator $\eta$ is an upper closure operator ${ }^{3}$ which saturates a set of sequences $S$ by adding new ${ }^{3} S \subseteq \eta(S)$ holds by definition, and it is easy to see that $\eta(\eta(S))=$ $\eta(S)$ holds and that $S \subseteq S^{\prime}$ implies $\eta(S) \subseteq \eta\left(S^{\prime}\right)$. sequences where redundant assumptions can be removed: an assumptions $a$ (in $K_{i}$ ) can be removed if $a^{j}$ appears as a stable atom (in $H_{i}$ ). Once a stable atom is "consumed" for satisfying an assumption it is removed from (the sets of stable atoms of) all the tuples appearing in the sequence, to avoid multiple uses of the same atom. Note that stable atoms are considered without the index in the condition $C T \models c \wedge B_{i} \leftrightarrow c \wedge A_{i}$, while they are considered as indexed atoms in the removal operation $H_{i} \backslash W$. The reason for this slight complication is explained by the following example. Assume that we have the set $S$ consisting of the only sequence $\left\langle c, \emptyset,\left\{a^{1}\right\}, d\right\rangle\left\langle c^{\prime},\{a\},\left\{a^{1}, a^{2}\right\}, d^{\prime}\right\rangle$. Such a sequence indicates that at the second step we have an assumption $a$, while both at the first and at the second step we have produced a stable atom $a$, which has been indexed by 1 and 2 , respectively. In order to satisfy the assumption $a$ we can use either $a^{1}$ or $a^{2}$. However, depending on what indexed atom we use, we obtain two different simplified sequences in $\eta(S)$, namely $\langle c, \emptyset, \emptyset, d\rangle\left\langle c^{\prime}, \emptyset,\left\{a^{2}\right\}, d^{\prime}\right\rangle$ and $\left\langle c, \emptyset,\left\{a^{1}\right\}, d\right\rangle\left\langle c^{\prime}, \emptyset,\left\{a^{1}\right\}, d^{\prime}\right\rangle$, which describes correctly the two different situations.

Before defining the composition operator $\|$ on sequences we need a notation for the sequences in $\mathcal{D}$ analogous to that one introduced for sequences of derivation steps:

Let $\sigma=\left\langle c_{1}, K_{1}, H_{1}, d_{1}\right\rangle\left\langle c_{2}, K_{2}, H_{2}, d_{2}\right\rangle \cdots\left\langle c_{n}, \emptyset, H_{n}, d_{n}\right\rangle \in$ $\mathcal{D}$ be a sequence for the goal $G$. We define

$V_{i n g}(\sigma)=F v(G)$ (the free variables of the goal $G$ ),

$V_{a s s}(\sigma)=\bigcup_{i=1}^{n-1} F v\left(K_{i}\right)$ (the variables in the assumptions of $\sigma)$

$V_{\text {stable }}(\sigma)=F v\left(H_{n}\right)=\bigcup_{i=1}^{n} F v\left(H_{i}\right)$ (the variables in the stable multisets of $\sigma$ ),

$V_{\text {constr }}(\sigma)=\bigcup_{i=1}^{n-1} F v\left(d_{i}\right) \backslash F v\left(c_{i}\right)$ (the variables in the output constraints of $\sigma$ which are not in the corresponding input constraints),

$$
V_{\text {loc }}(\sigma)=\left(V_{\text {constr }}(\sigma) \cup V_{\text {stable }}(\sigma)\right) \backslash\left(V_{\text {ass }}(\sigma) \cup V_{\text {ing }}(\sigma)\right) \text {. }
$$

We can now define the composition operator $\|$ on sequences. To simplify the notation we denote by $\|$ both the operator acting on sequences and that one acting on sets of sequences.

Definition 9. The operator $\|: \mathcal{D} \times \mathcal{D} \rightarrow \wp(\mathcal{D})$ is defined inductively as follows. Assume that $\sigma_{1}=\left\langle c_{1}, K_{1}, H_{1}, d_{1}\right\rangle \cdot \sigma_{1}^{\prime}$ and $\sigma_{2}=\left\langle c_{2}, K_{2}, H_{2}, d_{2}\right\rangle \cdot \sigma_{2}^{\prime}$ are sequences for the goals $G_{1}$ and $G_{2}$, respectively. If

$\left(V_{l o c}\left(\sigma_{1}\right) \cup F v\left(G_{1}\right)\right) \cap\left(V_{l o c}\left(\sigma_{2}\right) \cup F v\left(G_{2}\right)\right)=F v\left(G_{1}\right) \cap F v\left(G_{2}\right)$ then $\sigma_{1} \| \sigma_{2}$ is defined by cases as follows:

1. If both $\sigma_{1}$ and $\sigma_{2}$ have length 1 and have the same store, say $\sigma_{1}=\left\langle c, \emptyset, H_{1}, c\right\rangle$ and $\sigma_{2}=\left\langle c, \emptyset, H_{2}, c\right\rangle$, then

$$
\sigma_{1} \| \sigma_{2}=\left\{\left\langle c, \emptyset, H_{1} \uplus H_{2}, c\right\rangle\right\} .
$$

2. If $\sigma_{2}$ has length 1 and $\sigma_{1}$ has length $>1$ then

$$
\begin{array}{r}
\sigma_{1} \| \sigma_{2}=\left\{\left\langle c_{1}, K_{1}, H_{1} \uplus H_{2}, d_{1}\right\rangle \cdot \sigma \mid\right. \\
\sigma \in \sigma_{1}^{\prime} \| \sigma_{2} \text { and } \\
\left.C T \models \text { instore }(\sigma) \rightarrow d_{1}\right\} .
\end{array}
$$

The symmetric case is analogous and therefore omitted. 
3. If both $\sigma_{1}$ and $\sigma_{2}$ have length $>1$ then $\sigma_{1} \| \sigma_{2}=$

$$
\begin{aligned}
& \left\{\left\langle c_{1}, K_{1}, H_{1} \uplus H_{2}, d_{1}\right\rangle \cdot \sigma \in \mathcal{D} \mid \quad \sigma \in\left(\sigma_{1}^{\prime} \| \sigma_{2}\right)\right. \\
& \left.\quad \text { and } C T \models \text { instore }(\sigma) \rightarrow d_{1}\right\} \\
& \cup \\
& \left\{\left\langle c_{2}, K_{2}, H_{1} \uplus H_{2}, d_{2}\right\rangle \cdot \sigma \in \mathcal{D} \mid \quad \sigma \in\left(\sigma_{1} \| \sigma_{2}^{\prime}\right)\right. \\
& \left.\quad \text { and } C T \models \text { instore }(\sigma) \rightarrow d_{2}\right\}
\end{aligned}
$$

Finally the composition of sets of sequences $\|: \wp(\mathcal{D}) \times \wp(\mathcal{D}) \rightarrow$ $\wp(\mathcal{D})$ is defined by $S_{1} \| S_{2}=$

$$
\begin{aligned}
& \left\{\sigma \in \mathcal{D} \mid \text { there exist } \sigma_{1} \in S_{1} \text { and } \sigma_{2} \in S_{2}\right. \text { such that } \\
& \sigma=\left\langle c_{1}, K_{1}, H_{1}, d_{1}\right\rangle \cdots\left\langle c_{n}, \emptyset, H_{n}, c_{n}\right\rangle \in \eta\left(\sigma_{1} \| \sigma_{2}\right), \\
& \quad\left(V_{l o c}\left(\sigma_{1}\right) \cup V_{l o c}\left(\sigma_{2}\right)\right) \cap V_{a s s}(\sigma)=\emptyset \text { and for } i \in[1, n] \\
& \quad\left(V_{l o c}\left(\sigma_{1}\right) \cup V_{l o c}\left(\sigma_{2}\right)\right) \cap F v\left(c_{i}\right) \subseteq \\
& \left.\bigcup_{j=1}^{i-1} F v\left(d_{j}\right) \cup \bigcup_{j=1}^{i} F v\left(H_{j}\right)\right\} .
\end{aligned}
$$

Using this notion of composition of sequences we can show that the semantics $\mathcal{S}_{P}$ is compositional. Before proving the compositionality theorem we need some technical lemmas whose proof is included in the appendix.

Lemma 1. Let $G$ be a goal, $\delta \in \mathcal{S}_{P}^{\prime}(G)$ and let $\sigma=\alpha(\delta)$. Then $V_{r}(\delta)=V_{r}(\sigma)$ holds, where $r \in\{$ ing, ass, stable, constr, loc $\}$.

LEMMA 2. Let $P$ be a program, $G_{1}$ and $G_{2}$ be two goals and assume that $\delta \in \mathcal{S}_{P}^{\prime}\left(G_{1}, G_{2}\right)$. Then there exists $\delta_{1} \in \mathcal{S}_{P}^{\prime}\left(G_{1}\right)$ and $\delta_{2} \in \mathcal{S}_{P}^{\prime}\left(G_{2}\right)$, such that $\alpha(\delta) \in \eta\left(\alpha\left(\delta_{1}\right) \| \alpha\left(\delta_{2}\right)\right)$.

Lemma 3. Let $P$ be a program, let $G_{1}$ and $G_{2}$ be two goals and assume that $\delta_{1} \in \mathcal{S}_{P}^{\prime}\left(G_{1}\right)$ and $\delta_{2} \in \mathcal{S}_{P}^{\prime}\left(G_{2}\right)$ are two sequences such that the following hold:

\section{1. $\alpha\left(\delta_{1}\right) \| \alpha\left(\delta_{2}\right)$ is defined,}

2. $\sigma=\left\langle c_{1}, K_{1}, W_{1}, d_{1}\right\rangle \cdots\left\langle c_{n}, \emptyset, W_{n}, c_{n}\right\rangle \in \eta\left(\alpha\left(\delta_{1}\right) \| \alpha\left(\delta_{2}\right)\right)$,

3. $\left(V_{l o c}\left(\alpha\left(\delta_{1}\right)\right) \cup V_{l o c}\left(\alpha\left(\delta_{2}\right)\right)\right) \cap V_{a s s}(\sigma)=\emptyset$,

4. for $i \in[1, n],\left(V_{l o c}\left(\alpha\left(\delta_{1}\right)\right) \cup V_{l o c}\left(\alpha\left(\delta_{2}\right)\right)\right) \cap F v\left(c_{i}\right) \subseteq$ $\bigcup_{j=1}^{i-1} F v\left(d_{j}\right) \cup \bigcup_{j=1}^{i} F v\left(W_{j}\right)$.

Then there exists $\delta \in \mathcal{S}_{P}^{\prime}\left(G_{1}, G_{2}\right)$ such that $V_{\text {loc }}(\delta) \subseteq V_{l o c}\left(\delta_{1}\right) \cup$ $V_{\text {loc }}\left(\delta_{2}\right)$ and $\sigma=\alpha(\delta)$.

By using the above results we can prove the following theorem.

Theorem 1 (COMPositionality). Let $P$ be a program and let $G_{1}$ and $G_{2}$ be two goals. Then

$$
\mathcal{S}_{P}\left(G_{1}, G_{2}\right)=\mathcal{S}_{P}\left(G_{1}\right) \| \mathcal{S}_{P}\left(G_{2}\right)
$$

Proof We prove the two inclusions separately.

$\left.\left(\mathcal{S}_{P}\left(G_{1}, G_{2}\right) \subseteq \mathcal{S}_{P}\left(G_{1}\right)\right) \| \mathcal{S}_{P}\left(G_{2}\right)\right)$.] Let $\sigma \in \mathcal{S}_{P}\left(G_{1}, G_{2}\right)$. By definition of $\mathcal{S}_{P}$, there exists $\delta \in \mathcal{S}_{P}^{\prime}\left(G_{1}, G_{2}\right)$ such that $\sigma=$ $\alpha(\delta)$. By Lemma 2 there exist $\delta_{1} \in \mathcal{S}_{P}^{\prime}\left(G_{1}\right)$ and $\delta_{2} \in \mathcal{S}_{P}^{\prime}\left(G_{2}\right)$ such that $\sigma \in \eta\left(\alpha\left(\delta_{1}\right) \| \alpha\left(\delta_{2}\right)\right)$ and for $i=1,2, V_{l o c}\left(\delta_{i}\right) \subseteq$ $V_{l o c}(\alpha(\delta))$.

Let $\delta=\left\langle\left(G_{1}, G_{2}\right), c_{1}, K_{1}, B_{2}, d_{1}\right\rangle \cdots\left\langle B_{n}, c_{n}, \emptyset, B_{n}, c_{n}\right\rangle$ and let $\sigma=\left\langle c_{1}, K_{1}, W_{1}, d_{1}\right\rangle \cdots\left\langle c_{n}, \emptyset, W_{n}, c_{n}\right\rangle$, where $W_{n}=B_{n}$. Then in order to prove the thesis we have only to show that

$\left(V_{l o c}\left(\alpha\left(\delta_{1}\right)\right) \cup V_{l o c}\left(\alpha\left(\delta_{2}\right)\right)\right) \cap V_{a s s}(\sigma)=\emptyset$ and, for $i \in[1, n]$, $\left(V_{l o c}\left(\alpha\left(\delta_{1}\right)\right) \cup V_{l o c}\left(\alpha\left(\delta_{2}\right)\right)\right) \cap F v\left(c_{i}\right) \subseteq$ $\bigcup_{j=1}^{i-1} F v\left(d_{j}\right) \cup \bigcup_{j=1}^{i} F v\left(W_{j}\right)$.

First observe that by Lemma 1 and by hypothesis, we have that $V_{a s s}(\sigma)=V_{a s s}(\delta)$ and for $i=1,2$,

$$
V_{l o c}\left(\alpha\left(\delta_{i}\right)\right)=V_{l o c}\left(\delta_{i}\right) \subseteq V_{l o c}(\delta) .
$$

Then by the previous results and by the properties of the derivations $\left(V_{l o c}\left(\alpha\left(\delta_{1}\right)\right) \cup V_{l o c}\left(\alpha\left(\delta_{2}\right)\right)\right) \cap V_{a s s}(\sigma) \subseteq V_{l o c}(\delta) \cap V_{a s s}(\sigma)=\emptyset$. Moreover for $i \in[1, n]$,

$$
\begin{aligned}
& \left(V_{l o c}\left(\alpha\left(\delta_{1}\right)\right) \cup V_{l o c}\left(\alpha\left(\delta_{2}\right)\right)\right) \cap F v\left(c_{i}\right) \subseteq \\
& V_{l o c}(\delta) \cap F v\left(c_{i}\right) \subseteq \bigcup_{j=1}^{i-1} F v\left(d_{j}\right) \cup V_{\text {stable }}(\delta)
\end{aligned}
$$

hold. Now, observe that if $x \in V_{l o c}(\delta) \cap F v\left(c_{i}\right) \cap V_{\text {stable }}(\delta)$, then $x \in \bigcup_{j=1}^{i} F v\left(B_{j}\right) \cap V_{\text {stable }}(\delta)$ and then $x \in \bigcup_{j=1}^{i} F v\left(W_{j}\right)$ and this completes the proof of the first inclusion.

$\left(\mathcal{S}_{P}\left(G_{1}, G_{2}\right) \supseteq \mathcal{S}_{P}\left(G_{1}\right) \| \mathcal{S}_{P}\left(G_{2}\right)\right)$. Let $\sigma \in \mathcal{S}_{P}\left(G_{1}\right)$ $\mathcal{S}_{P}\left(G_{2}\right)$. By definition of $\mathcal{S}_{P}$ and of $\|$ there exist $\delta_{1} \in \mathcal{S}_{P}^{\prime}\left(G_{1}\right)$ and $\delta_{2} \in \mathcal{S}_{P}^{\prime}\left(G_{2}\right)$, such that $\sigma_{1}=\alpha\left(\delta_{1}\right), \sigma_{2}=\alpha\left(\delta_{2}\right), \sigma_{1} \| \sigma_{2}$ is defined, $\sigma=\left\langle c_{1}, K_{1}, H_{1}, d_{1}\right\rangle \cdots\left\langle c_{n}, \emptyset, H_{n}, c_{n}\right\rangle \in \eta\left(\sigma_{1} \|\right.$ $\left.\sigma_{2}\right),\left(V_{l o c}\left(\sigma_{1}\right) \cup V_{l o c}\left(\sigma_{2}\right)\right) \cap V_{a s s}(\sigma)=\emptyset$ and for $i \in[1, n]$, $\left(V_{l o c}\left(\sigma_{1}\right) \cup V_{l o c}\left(\sigma_{2}\right)\right) \cap F v\left(c_{i}\right) \subseteq \bigcup_{j=1}^{i-1} F v\left(d_{j}\right) \cup \bigcup_{j=1}^{i} F v\left(H_{j}\right)$. The proof is then straightforward by using Lemma 3 .

\subsection{Correctness}

In order to show the correctness of the semantics $\mathcal{S}_{P}$ w.r.t. the (input/output) observables $\mathcal{S} \mathcal{A}_{P}$, we first introduce a different characterization of $\mathcal{S} \mathcal{A}_{P}$ obtained by using the new transition system defined in Table 2.

Definition 10. Let $P$ be a program and let $G$ be a goal and let $\longrightarrow$ be (the least relation) defined by the rules in Table 2 . We define

$$
\mathcal{S A}^{\prime}{ }_{P}(G)=\left\{\exists_{-F v(G)} c \mid\langle G, \emptyset\rangle \longrightarrow_{P}^{*}\langle\emptyset, c\rangle \longrightarrow_{P}\right\} .
$$

The correspondence of $\mathcal{S} \mathcal{A}^{\prime}$ with the original notion $\mathcal{S} \mathcal{A}$ is stated by the following proposition, whose proof is immediate.

Proposition 1. Let $P$ be a program and let $G$ be a goal. Then

$$
\mathcal{S} \mathcal{A}_{P}(G)=\mathcal{S A}_{P}^{\prime}(G) \text {. }
$$

The observables $\mathcal{S} \mathcal{A}_{P}^{\prime}$, and therefore $\mathcal{S} \mathcal{A}_{P}$, describing answers of successful computations can be obtained from $\mathcal{S}$ by considering suitable sequences, namely those sequences which do not perform assumptions neither on CHR constraints nor on built-in constraints. The first condition means that the second components of tuples must be empty, while the second one means that the assumed constraint at step i must be equal to the produced constraint of steps i-1. We call "connected" those sequences which satisfy these requirements: 
Definition 11. (Connected sequences) Assume that

$$
\sigma=\left\langle c_{1}, K_{1}, H_{1}, d_{1}\right\rangle \ldots\left\langle c_{n}, K_{n}, H_{n}, c_{n}\right\rangle
$$

is a sequence in $\mathcal{D}$. We say that $\sigma$ is connected if $K_{j}=\emptyset$ for each $j, 1 \leq j \leq n$ and $d_{i}=c_{i+1}$, for each $i, 1 \leq i \leq n-1$.

The proof of the following result derives from the definition of connected sequence and an easy inductive argument. Given a sequence $\sigma=\left\langle c_{1}, K_{1}, H_{1}, d_{1}\right\rangle \ldots\left\langle c_{n}, K_{n}, H_{n}, d_{n}\right\rangle$, we denote by $\operatorname{store}(\sigma)$ the built-in constraint $d_{n}$, by $\operatorname{result}(\sigma)$ the constraint $d_{n} \wedge H_{n}$ and by $\operatorname{lastg}(\sigma)$ the goal $H_{n}$.

PROPOSITION 2. Let $P$ be a program and let $G$ be a goal. Then $\mathcal{S} \mathcal{A}_{P}^{\prime}(G)=$

$\left\{\exists_{-F v(G)} c \mid\right.$ there exists $\sigma \in \mathcal{S}_{P}(G)$ such that instore $(\sigma)=\emptyset$, $\sigma$ is connected, $\operatorname{lastg}(\sigma)=\emptyset$ and $c=\operatorname{store}(\sigma)\}$.

The following corollary is immediate from Proposition 1.

Corollary 1 (CORRECTNESS). Let $P$ be a program and let $G$ be a goal. Then $\mathcal{S A}_{P}(G)=$

$\left\{\exists_{-F v(G)} c \mid\right.$ there exists $\sigma \in \mathcal{S}_{P}(G)$ such that instore $(\sigma)=\emptyset$, $\sigma$ is connected, $\operatorname{lastg}(\sigma)=\emptyset$ and $c=$ store $(\sigma)\}$.

\section{A MORE REFINED SEMANTICS}

As previously mentioned, the operational semantics that we have considered in this paper is somehow naive: In fact, since propagation rules do not remove user defined constraints (see rule Propagate in Table 1), when a propagate rule is applied it introduces an additional infinite computation (obtained by subsequent applications of the same rule). Of course, as previously mentioned, the terminating computations are not affected, as the application of a simplification rule after a propagation rule can cause the termination of the computation.

In [1] it has been defined a more refined operational semantics which avoid these infinite computations by allowing to apply at most once a propagation rule to the same constraint. Essentially the idea is to memorize in a "token store", to be added to the global state, some "tokens" containing the information about which propagation rules can be applied to a given set of user-defined constraints. Each "token" consists of a propagation rule name and of the set of candidate constraints for that rule. A propagation rule can then be applied only if the store contains the appropriate token.

We could take into account this refined operational semantics by using a slight extension of our semantic construction. More precisely, we first consider "concrete" sequences consisting of tuples of the form $\left\langle G, c, T, K, G^{\prime}, T^{\prime}, d\right\rangle$, where $T$ and $T^{\prime}$ are token stores as defined in [1]. Such a tuple represents exactly a derivation step $\langle G, c, T\rangle \longrightarrow^{K}\left\langle G^{\prime}, d, T^{\prime}\right\rangle$, according to the operational semantics in [1]. The sequences we consider are terminated by tuples of the form $\langle G, c, T, \emptyset, G, c, T\rangle$, which represent a terminating step. Since a sequence represents a derivation, we assume that the "output" goal $G^{\prime}$ and token store $T^{\prime}$ at step $i$ is equal to the "input" goal $G$ and to the token store $T$ at step $i+1$, respectively. From these concrete sequences we extract the same abstract sequences which are the objects of our semantic domain: From each tuple $\left\langle G, c, T, K, G^{\prime}, d, T^{\prime}\right\rangle$ in a concrete sequence $\delta$ we extract a tuple of the form $\langle c, K, H, d\rangle$ where we consider as before the input and output store ( $c$ and $d$, respectively) and the assumptions $(K)$, while we do not consider anymore the output goal $G^{\prime}$ and the token stores $T$ and $T^{\prime}$. The abstraction operator which extracts from the concrete sequences the sequences used in the semantic domain is a simple extension to that one given in Definition 5. In order to obtain a compositionality result we then define how two sequences describing a computation of $A$ and $B$ according to this refined operational semantics, respectively, can be composed in order to obtain a computation of $A, B$. Such a composition is defined by a (semantic) operator, which performs an interleaving of the actions described by the two sequences. This new operator is similar to that one defined in Definition 9 even though the technicalities are different.

Recently a more refined semantics has been defined in [10] in order to describe precisely the operational semantics implicitly used by (Prolog) implementations of CHR. Although this refined operational semantics is still non-deterministic, the order in which transitions are applied and the order in which occurrences are visited are decided. This semantics is therefore substantially different from the one we consider and apparently it is difficult to give a compositional characterization for it.

\section{CONCLUSIONS}

In this paper we have introduced a semantics for CHR which is compositional w.r.t. the and-composition of goals and which is correct w.r.t "success answers", a notion of observable which considers the results of successful computations where all the user-defined constraints have been rewritten into built-in constraints. We are not aware of other compositional characterizations of CHR answers and only [14] addresses compositionality of CHR rules (but only for a subset of CHR). Our work can be considered as a first step which can be extended along several different lines.

Firstly, it would be desirable to obtain a compositional characterization also for "qualified answers" obtained by considering computations terminating with a user-defined constraint which does not need to be empty (see Definition 3 ). This could be done by a slight extension of our model: The problem here is that, given a tuple $\left\langle G, c, K, G^{\prime}, d\right\rangle$, in order to reconstruct correctly the qualified answers we need to know whether the configuration $\left\langle G^{\prime}, d\right\rangle$ is terminating or not (that is, if $\left\langle G^{\prime}, d\right\rangle \not \rightarrow$ holds). This could be solved by introducing some termination modes, at the price of a further complication of the traces used in our semantics. Also, as previously mentioned, we are currently extending our semantics in order do describe the more refined operational semantics given in [1].

A second possible extension is the investigation of the full abstraction issue. For obvious reasons it would be desirable to introduce in the semantics the minimum amount of information needed to obtain compositionality, while preserving correctness. In other terms, one would like to obtain a results of this kind: $\mathcal{S}_{P}(G)=\mathcal{S}_{P}\left(G^{\prime}\right)$ if and only if, for any $H, \mathcal{S A}_{P}(G, H)=\mathcal{S} \mathcal{A}_{P}\left(G^{\prime}, H\right)$ (our Corollary 1 only ensures that the "only if" part holds). Such a full abstraction result could be difficult to achieve, however techniques similar to those used in $[6,3]$ for analogous results in the context of ccp could be considered

It would be interesting also to study further notions of compositionality, for example that one which considers union of program rules rather than conjunctions of goals, analogously to what has 
been done in [7]. However, due to the presence of synchronization, the simple model based on clauses defined in [7] cannot be used for CHR.

As mentioned in the introduction, the main interest related to a compositional semantics in the possibility to provide a basis to define compositional analysis and verification tools. In our case, it would be interesting to investigate to what extent the compositional proof systems à la Hoare defined in [2, 4] for (timed) ccp languages, based on resting points and trace semantics, can be adapted to the case of CHR.

Acknowledgments We thank Michael Maher for having initially suggested the problem of compositionality for CHR semantics.

\section{REFERENCES}

[1] S. Abdennadher. Operational semantics and confluence of constraint propagation rules. In G. Smolka. editor, Proc. Third Int'l Conf. on Principles and Practice of Constraint Programming (CP 97), Lecture Notes in Computer Science 1330. Springer-Verlag, 1997.

[2] F.S. de Boer, M. Gabbrielli, E. Marchiori and C. Palamidessi. Proving Concurrent Constraint Programs Correct. Transactions on Programming Languages and Systems (TOPLAS), 19(5): 685-725. ACM Press, 1997.

[3] F.S. de Boer, M. Gabbrielli, and M.C. Meo. Semantics and expressive power of a timed concurrent constraint language. In G. Smolka. editor, Proc. Third Int'l Conf. on Principles and Practice of Constraint Programming (CP 97), Lecture Notes in Computer Science. Springer-Verlag, 1997.

[4] F.S. de Boer, M. Gabbrielli and M.C. Meo. Proving correctness of Timed Concurrent Constraint Programs. ACM Transactions on Computational Logic. To appear.

[5] F.S. de Boer, J.N. Kok, C. Palamidessi, and J.J.M.M. Rutten. The failure of failures in a paradigm for asynchronous communication. In J.C.M. Baeten and J.F. Groote, editors, Proceedings of CONCUR'91, vol. 527 of LNCS, pages 111-126. Springer-Verlag, 1991.

[6] F.S. de Boer and C. Palamidessi. A Fully Abstract Model for Concurrent Constraint Programming. In S. Abramsky and T.S.E. Maibaum, editors, Proc. of TAPSOFT/CAAP, vol. 493 of LNCS, pages 296-319. Springer-Verlag, 1991.

[7] A. Bossi, M. Gabbrielli, G. Levi, and M. C. Meo. A Compositional Semantics for Logic Programs. Theoretical Computer Science 122(1-2): 3-47, 1994.

[8] S. Brookes. A fully abstract semantics of a shared variable parallel language. In Proc. Eighth IEEE Symposium on Logic In Computer Science. IEEE Computer Society Press, 1993.

[9] B.A. Davey and H.A. Priestley. Introduction to Lattices and Order. Cambridge University Press, 1990.

[10] Gregory J. Duck, Maria Garcia de la Banda, Peter J. Stuckey. The Refined Operational Semantics of Constraint Handling Rules. in Proc. of the 20th International Conference on Logic Programming, (ICLP'04), 2004.

[11] T. Früwirth. Introducing simplification rules. TR ECRC-LP-63, ECRC Munich. October 1991.
[12] T. Früwirth. Theory and practice of Constraint Handling Rules. Journal of Logic Programming, 1994:19, 20:1-679.

[13] B. Jonsson. A model and a proof system for asynchronous processes. In Proc. of the 4th ACM Symp. on Principles of Distributed Computing, pages 49-58. ACM Press, 1985.

[14] M. Maher. Propagation Completeness of Reactive Constraints. In Proc. International Conference on Logic Programming (ICLP), 148 - 162, 2002.

[15] V.A. Saraswat and M. Rinard. Concurrent constraint programming. In Proc. of POPL, pages 232-245. ACM Press, 1990.

[16] V.A. Saraswat, M. Rinard, and P. Panangaden. Semantics foundations of Concurrent Constraint Programming. In Proc. of POPL. ACM Press, 1991. 


\section{APPENDIX}

In this appendix we provide the proofs of some lemmas used in the paper. The appendix is included only for the convenience of the reviewers and it is not meant to be part of the paper.

Lemma 4. (Lemma 1) Let $G$ be a goal, $\delta \in \mathcal{S}_{P}^{\prime}(G)$ and let $\sigma=\alpha(\delta)$. Then

$V_{r}(\delta)=V_{r}(\sigma)$, where $r \in\{$ ing, ass, stable, constr, loc $\}$.

Proof If $r \in\{$ ing, ass, stable, constr $\}$ then the proof is straightforward by definition of $\alpha$ and of $V_{r}$. Then we have only to prove that $V_{l o c}(\delta)=V_{l o c}(\sigma)$.

The proof is by induction on $n=$ lenght $(\delta)$.

$n=1$ ) In this case $\delta=\langle G, c, \emptyset, G, c\rangle, \sigma=\langle c, \emptyset, G, c\rangle$, and therefore, by definition $V_{l o c}(\delta)=V_{l o c}(\sigma)=\emptyset$.

$n \geq 1)$ Let $\delta=\left\langle G_{1}, c_{1}, K_{1}, G_{2}, d_{1}\right\rangle\left\langle G_{2}, c_{2}, K_{2}, G_{3}, d_{3}\right\rangle \cdots$

$\left\langle G_{n}, c_{n}, \emptyset, G_{n}, c_{n}\right\rangle$, where $G=G_{1}$.

By definition of $\mathcal{S}_{P}^{\prime}(G)$, there exists $\delta^{\prime} \in \mathcal{S}_{P}^{\prime}\left(G_{2}\right)$ such that $t=\left\langle G_{1}, c_{1}, K_{1}, G_{2}, d_{1}\right\rangle$ is compatible with $\delta^{\prime}$ and $\delta=t \cdot \delta^{\prime}$. By inductive hypothesis, we have that $V_{l o c}\left(\delta^{\prime}\right)=V_{l o c}\left(\sigma^{\prime}\right)$, where $\sigma^{\prime}=\alpha\left(\delta^{\prime}\right)$.

Moreover, by definition of $\alpha, \sigma=\left\langle c_{1}, K_{1}, H_{1}, d_{1}\right\rangle \cdot \sigma^{\prime}$, where $H_{1}$ is the multiset consisting of all the atoms in $G_{1}$ which are stable in $\delta$.

By definition of $V_{l o c}$ and by inductive hypothesis

$$
\begin{aligned}
V_{l o c}(\delta) & =\bigcup_{i=1}^{n-1} F v\left(G_{i+1}, d_{i}\right) \backslash F v\left(G_{i}, c_{i}, K_{i}\right) \\
& =V_{l o c}\left(\delta^{\prime}\right) \cup\left(F v\left(G_{2}, d_{1}\right) \backslash F v\left(G_{1}, c_{1}, K_{1}\right)\right) \\
& =V_{l o c}\left(\sigma^{\prime}\right) \cup\left(F v\left(G_{2}, d_{1}\right) \backslash F v\left(G_{1}, c_{1}, K_{1}\right)\right) .(2)
\end{aligned}
$$

Moreover, since $V_{\text {stable }}(\sigma)=V_{\text {stable }}\left(\sigma^{\prime}\right)$ and by definition of $V_{l o c}$, we have that

$V_{\text {loc }}\left(\sigma^{\prime}\right)=\left(V_{\text {constr }}\left(\sigma^{\prime}\right) \cup V_{\text {stable }}(\sigma)\right) \backslash\left(V_{\text {ass }}\left(\sigma^{\prime}\right) \cup F v\left(G_{2}\right)\right)$.

Therefore by (2), by properties of $\cup$ and since $F v\left(G_{2}\right) \cap$ $F v\left(G_{1}, c_{1}, K_{1}\right) \subseteq F v\left(G_{2}\right) \cap F v\left(G_{1}\right)$, we have that

$V_{l o c}(\delta)=$

$$
\begin{aligned}
& \left(\left(V_{\text {constr }}\left(\sigma^{\prime}\right) \cup V_{\text {stable }}(\sigma)\right) \backslash\left(V_{\text {ass }}\left(\sigma^{\prime}\right) \cup F v\left(G_{2}\right)\right)\right) \cup \\
& \left(F v\left(G_{2}\right) \backslash F v\left(G_{1}\right)\right) \cup\left(F v\left(d_{1}\right) \backslash F v\left(G_{1}, c_{1}, K_{1}\right)\right) . \text { (3) }
\end{aligned}
$$

By properties of $\cup$, we have that

$$
\begin{aligned}
& \left(\left(V_{\text {constr }}\left(\sigma^{\prime}\right) \cup V_{\text {stable }}(\sigma)\right) \backslash\left(V_{\text {ass }}\left(\sigma^{\prime}\right) \cup F v\left(G_{2}\right)\right)\right) \\
& \cup\left(F v\left(G_{2}\right) \backslash F v\left(G_{1}\right)\right)= \\
& \left(\left(V_{\text {constr }}\left(\sigma^{\prime}\right) \cup V_{\text {stable }}(\sigma)\right) \backslash\right. \\
& \left.\left(V_{\text {ass }}\left(\sigma^{\prime}\right) \cup\left(F v\left(G_{2}\right) \cap F v\left(G_{1}\right)\right)\right)\right) \\
& \cup\left(F v\left(G_{2}\right) \backslash F v\left(G_{1}\right)\right) .
\end{aligned}
$$

Now let $x \in F v\left(G_{1}\right) \backslash F v\left(G_{2}\right)$. Then by definition $x \in$ $F v(t)$, since $t$ is compatible with $\delta^{\prime}$ and by point 2) of definition of compatibility, we have that $x \notin V_{l o c}\left(\delta^{\prime}\right)$. Then following holds.

- Assume that $x \in V_{\text {constr }}\left(\sigma^{\prime}\right)=V_{\text {constr }}\left(\delta^{\prime}\right)$. Then since $x \notin V_{l o c}\left(\delta^{\prime}\right)$ and since $x \notin F v\left(G_{2}\right)$, we have that there exists $i \in[2, n-1]$ such that $x \in F v\left(K_{i}\right)$ and therefore $x \in V_{\text {ass }}\left(\delta^{\prime}\right)=V_{\text {ass }}\left(\sigma^{\prime}\right)$.
- $x \notin V_{\text {stable }}(\sigma)=V_{\text {stable }}\left(\sigma^{\prime}\right)=V_{\text {stable }}\left(\delta^{\prime}\right)$ since $x \notin$ $F v\left(G_{2}\right)$ and $x \notin V_{l o c}\left(\delta^{\prime}\right)$.

By the previous results and by (3) and (4), we have that

$$
\begin{aligned}
& V_{\text {loc }}(\delta)= \\
& \left(\left(V_{\text {constr }}\left(\sigma^{\prime}\right) \cup V_{\text {stable }}(\sigma)\right) \backslash\left(V_{\text {ass }}\left(\sigma^{\prime}\right) \cup F v\left(G_{1}\right)\right)\right) \cup \\
& \left(F v\left(G_{2}\right) \backslash F v\left(G_{1}\right)\right) \cup\left(F v\left(d_{1}\right) \backslash F v\left(G_{1}, c_{1}, K_{1}\right)\right) .(5)
\end{aligned}
$$

Now, let $x \in F v\left(K_{1}\right) \cap\left(V_{\text {constr }}\left(\sigma^{\prime}\right) \cup V_{\text {stable }}(\sigma)\right)$. If $x \notin$ $F v\left(G_{1}\right)$ then by definition of derivation step, we have that $x \notin F v\left(G_{2}\right)$. Then, by using an argument similar to the previous one, we have that $V_{a s s}\left(\sigma^{\prime}\right)$. Then, by (5),

$$
\begin{aligned}
& V_{\text {loc }}(\delta)= \\
& \quad\left(\left(V_{\text {constr }}\left(\sigma^{\prime}\right) \cup V_{\text {stable }}(\sigma)\right) \backslash\left(V_{\text {ass }}(\sigma) \cup F v\left(G_{1}\right)\right)\right) \cup \\
& \left(F v\left(G_{2}\right) \backslash F v\left(G_{1}\right)\right) \cup\left(F v\left(d_{1}\right) \backslash F v\left(G_{1}, c_{1}, K_{1}\right)\right) .(6)
\end{aligned}
$$

Now let $x \in\left(F v\left(d_{1}\right) \backslash F v\left(c_{1}\right)\right) \cap V_{\text {ass }}\left(\sigma^{\prime}\right)$. Since by point 4) of definition of compatibility $V_{l o c}(t) \cap V_{a s s}\left(\sigma^{\prime}\right)=\emptyset$, we have that $x \in F v\left(G_{1}, K_{1}\right)$. Then

$$
\begin{array}{ll}
F v\left(d_{1}\right) \backslash F v\left(G_{1}, c_{1}, K_{1}\right) & = \\
\left(F v\left(d_{1}\right) \backslash F v\left(c_{1}\right)\right) \backslash F v\left(G_{1}, K_{1}\right) & = \\
\left(F v\left(d_{1}\right) \backslash F v\left(c_{1}\right)\right) \backslash\left(F v\left(G_{1}, K_{1}\right) \cup V_{a s s}\left(\sigma^{\prime}\right)\right) & = \\
\left(F v\left(d_{1}\right) \backslash F v\left(c_{1}\right)\right) \backslash\left(F v\left(G_{1}\right) \cup V_{a s s}(\sigma)\right) . &
\end{array}
$$

Then by (6),

$$
\begin{aligned}
& V_{l o c}(\delta)= \\
& \quad\left(\left(V_{\text {constr }}(\sigma) \cup V_{\text {stable }}(\sigma)\right) \backslash\left(V_{\text {ass }}(\sigma) \cup F v\left(G_{1}\right)\right)\right) \\
& \quad \cup\left(F v\left(G_{2}\right) \backslash F v\left(G_{1}\right)\right) .
\end{aligned}
$$

Finally observe that if $x \in F v\left(G_{2}\right) \backslash F v\left(G_{1}\right)$, then by definition of derivation step, $x \in V_{l o c}(t)$ and therefore, by definition of compatibility, $x \notin V_{a s s}(\sigma)$ and by point 3) of definition of compatibility, $x \in V_{\text {constr }}(\sigma) \cup V_{\text {stable }}(\sigma)$. Then by (7), by the previous result and by definition of $V_{l o c}$,

$$
\begin{array}{ll}
V_{\text {loc }}(\delta) & = \\
\left(\left(V_{\text {constr }}(\sigma) \cup V_{\text {stable }}(\sigma)\right) \backslash\left(V_{\text {ass }}(\sigma) \cup F v\left(G_{1}\right)\right)\right) & = \\
V_{\text {loc }}(\sigma) &
\end{array}
$$

and then the thesis holds.

In the following, given a sequence $\gamma$, where $\gamma \in \mathcal{S} e q \cup \mathcal{D}$, we will denote by $\operatorname{Inc}(\gamma)$ the set of input constraints of $\gamma$. Moreover, we will denote by $A s s(\gamma)$ and Stable $(\gamma)$ the multiset of assumptions of $\gamma$ and the multiset of atoms in the last goal of $\gamma$ respectively. Finally, given a sequence of derivation steps

$$
\delta=\left\langle B_{1}, c_{1}, K_{1}, B_{2}, d_{1}\right\rangle \ldots\left\langle B_{n}, c_{n}, \emptyset, B_{n}, c_{n}\right\rangle
$$

and a goal $W$, we denote by $\delta \oplus W$ the sequence $\left\langle\left(B_{1}, W\right), c_{1}, K_{1},\left(B_{2}, W\right), d_{1}\right\rangle \ldots\left\langle\left(B_{n}, W\right), c_{n}, \emptyset,\left(B_{n}, W\right), c_{n}\right\rangle$ and by $\delta \ominus W$ the sequence

$$
\left\langle B_{1} \backslash W, c_{1}, K_{1}, B_{2} \backslash W, d_{1}\right\rangle \ldots\left\langle B_{n} \backslash W, c_{n}, \emptyset, B_{n} \backslash W, c_{n}\right\rangle .
$$

Lemma 5. Let $P$ be a program and let $H$ and $G$ be two goals such that there exists a derivation step $s=\left\langle(H, G), c_{1}\right\rangle \longrightarrow K_{1}$ $\left\langle\left(H^{\prime}, G^{\prime}, B\right), d_{1}\right\rangle$, where $H=\left(H^{\prime}, H^{\prime \prime}\right), G=\left(G^{\prime}, G^{\prime \prime}\right)$ and $H^{\prime \prime} \neq \emptyset$. Assume that there exists $\delta \in \mathcal{S}_{P}^{\prime}(H, G)$ such that $\delta=t \cdot \delta^{\prime}$, where $t=\left\langle(H, G), c_{1}, K_{1},\left(H^{\prime}, G^{\prime}, B\right), d_{1}\right\rangle, \delta^{\prime} \in$ $\mathcal{S}_{P}^{\prime}\left(H^{\prime}, G^{\prime}, B\right)$ and $t$ is compatible with $\delta^{\prime}$. Moreover assume that there exists $\delta_{1}^{\prime} \in \mathcal{S}_{P}^{\prime}\left(H^{\prime}, B\right)$ and $\delta_{2}^{\prime} \in \mathcal{S}_{P}^{\prime}\left(G^{\prime}\right)$, such that 
- $\alpha\left(\delta^{\prime}\right) \in \eta\left(\alpha\left(\delta_{1}^{\prime}\right) \| \alpha\left(\delta_{2}^{\prime}\right)\right)$,

- for $i=1,2, V_{l o c}\left(\delta_{i}^{\prime}\right) \subseteq V_{l o c}\left(\delta^{\prime}\right)$,

- $\operatorname{Inc}\left(\delta_{1}^{\prime}\right) \subseteq \operatorname{Inc}\left(\delta^{\prime}\right)$,

- $\operatorname{Ass}\left(\delta_{1}^{\prime}\right) \subseteq \operatorname{Ass}\left(\delta^{\prime}\right) \uplus \operatorname{Stable}\left(\delta_{2}^{\prime}\right)$ and $\operatorname{Ass}\left(\delta_{2}^{\prime}\right) \subseteq \operatorname{Ass}\left(\delta^{\prime}\right) \uplus \operatorname{Stable}\left(\delta_{1}^{\prime}\right)$.

Then $\delta_{1}=t^{\prime} \cdot \delta_{1}^{\prime} \in \mathcal{S}_{P}^{\prime}(H)$, where $t^{\prime}=\left\langle H, c_{1}, K_{1},\left(H^{\prime}, B\right), d_{1}\right\rangle$, $\delta_{2}=\delta_{2}^{\prime} \oplus G^{\prime \prime} \in \mathcal{S}_{P}^{\prime}(G)$ and $\alpha\left(\delta_{1}\right) \| \alpha\left(\delta_{2}\right)$ is defined.

Proof First we prove that $t^{\prime} \cdot \delta_{1}^{\prime} \in \mathcal{S}_{P}^{\prime}(H)$. By definition of $\mathcal{S}_{P}^{\prime}$ and by construction, we have only to prove that $t^{\prime}$ is compatible with $\delta_{1}^{\prime}$.

1. By hypothesis $\operatorname{Inc}\left(\delta_{1}^{\prime}\right) \subseteq \operatorname{Inc}\left(\delta^{\prime}\right)$ and therefore

$$
C T \models \text { instore }\left(\delta_{1}^{\prime}\right) \rightarrow \text { instore }\left(\delta^{\prime}\right) .
$$

Moreover since $t$ is compatible with $\delta^{\prime}$, we have that $C T \models$ instore $\left(\delta^{\prime}\right) \rightarrow c_{1}$ and then the thesis.

2. By hypothesis $V_{l o c}\left(\delta_{1}^{\prime}\right) \subseteq V_{l o c}\left(\delta^{\prime}\right)$ and by construction $F v\left(t^{\prime}\right) \subseteq F v(t)$.

Then $V_{l o c}\left(\delta_{1}^{\prime}\right) \cap F v\left(t^{\prime}\right) \subseteq V_{l o c}\left(\delta^{\prime}\right) \cap F v(t)=\emptyset$, where the last equality follows since $t$ is compatible with $\delta^{\prime}$.

3. Assume that

$$
\begin{aligned}
& \delta_{1}^{\prime}=\left\langle\left(H^{\prime}, B\right), e_{1}, M_{1}, H_{2}, f_{1}\right\rangle \cdots\left\langle H_{l}, e_{l}, \emptyset, H_{l}, e_{l}\right\rangle \\
& \delta_{2}^{\prime}=\left\langle G^{\prime}, r_{1}, N_{1}, G_{2}^{\prime}, s_{1}\right\rangle \cdots\left\langle G_{m}^{\prime}, r_{m}, \emptyset, G_{m}^{\prime}, r_{m}\right\rangle \\
& \delta^{\prime}=\left\langle\left(H^{\prime}, G^{\prime}, B\right), c_{2}, K_{2}, R_{3}, d_{2}\right\rangle \cdots\left\langle R_{n}, c_{n}, \emptyset, R_{n}, c_{n}\right\rangle,
\end{aligned}
$$

where $e_{l}=r_{m}=c_{n}$. We have to prove that for $i \in[1, l]$, $V_{l o c}\left(t^{\prime}\right) \cap F v\left(e_{i}\right) \subseteq \bigcup_{j=1}^{i-1} F v\left(f_{j}\right) \cup F v\left(d_{1}\right) \cup V_{\text {stable }}\left(\delta_{1}^{\prime}\right)$. Let $x \in V_{l o c}\left(t^{\prime}\right) \cap F v\left(e_{i}\right)$, where $i \in[1, l]$.

By definition of $\|$, there exists $h \in[2, n]$ such that $e_{i}=c_{h}$. Therefore, since $V_{l o c}\left(t^{\prime}\right)=V_{l o c}(t)$ and $t$ is compatible with $\delta^{\prime}$, we have that

$$
x \in \bigcup_{j=1}^{h-1} F v\left(d_{j}\right) \cup V_{\text {stable }}\left(\delta^{\prime}\right) .
$$

Moreover, since $x \in V_{l o c}\left(t^{\prime}\right)$ and by hypothesis $V_{l o c}\left(\delta_{2}^{\prime}\right) \subseteq$ $V_{l o c}\left(\delta^{\prime}\right)$

$$
x \notin F v(G) \cap V_{l o c}\left(\delta^{\prime}\right) \supseteq F v\left(G^{\prime}\right) \cap V_{l o c}\left(\delta_{2}^{\prime}\right) .
$$

Now observe that given a derivation $\tilde{\delta}$, we have that

$$
V_{\text {Stable }}(\tilde{\delta}) \subseteq V_{\text {ing }}(\tilde{\delta}) \cup V_{l o c}(\tilde{\delta}) .
$$

\section{Therefore}

$$
V_{\text {stable }}\left(\delta^{\prime}\right) \subseteq
$$

(by definition of $\|$ and since by hypothesis $\left.\alpha\left(\delta^{\prime}\right) \in \eta\left(\alpha\left(\delta_{1}^{\prime}\right) \| \alpha\left(\delta_{2}^{\prime}\right)\right)\right)$

$$
V_{\text {stable }}\left(\delta_{1}^{\prime}\right) \cup V_{\text {stable }}\left(\delta_{2}^{\prime}\right) \subseteq
$$$$
\text { (by (10)) }
$$

$$
V_{\text {stable }}\left(\delta_{1}^{\prime}\right) \cup F v\left(G^{\prime}\right) \cup V_{\text {loc }}\left(\delta_{2}^{\prime}\right) \text {. }
$$

Then by (8) and (9), we have that

$$
x \in \bigcup_{j=1}^{h-1} F v\left(d_{j}\right) \cup V_{\text {stable }}\left(\delta_{1}^{\prime}\right) .
$$

Then to prove the thesis, we have to prove that if $x \in \bigcup_{j=1}^{h-1} F v\left(d_{j}\right) \cup V_{\text {stable }}\left(\delta_{1}^{\prime}\right)$ then $x \in \bigcup_{j=1}^{i-1} F v\left(f_{j}\right) \cup F v\left(d_{1}\right) \cup V_{\text {stable }}\left(\delta_{1}^{\prime}\right)$.
Let us to assume that $x \in \bigcup_{j=2}^{h-1} F v\left(d_{j}\right)$ and let $k$ the least index $j \in[2, h-1]$ such that $x \in F v\left(d_{j}\right)$.

If $d_{k}$ is an output constraint of $\delta_{1}^{\prime}$, i.e. there exists $j \in[1, i-1]$ such that $d_{k}=f_{j}$, the proof is terminated.

Now assume that $d_{k}$ is an output constraint of $\delta_{2}^{\prime}$, i.e. there exists $w \in[1, m]$ such that $d_{k}=s_{w}$ and for each $j \in[1, w-$ 1 , we have that $x \notin F v\left(s_{j}\right)$. Since $k$ is the least index $j$ such that $x \in F v\left(d_{j}\right)$ and since $t$ is compatible with $\delta^{\prime}$, we have that $x \notin F v\left(c_{k}\right)$ and therefore $x \notin F v\left(r_{w}\right)$.

Moreover, since by (9), $x \notin F v\left(G^{\prime}\right) \cup V_{l o c}\left(\delta_{2}^{\prime}\right)$, we have that $x \notin F v\left(G_{w}^{\prime}\right)$. Then by definition of derivation step, since $x \in F v\left(s_{w}\right) \backslash\left(F v\left(r_{w}\right) \cup F v\left(G_{w}^{\prime}\right)\right)$, we have that $x \in$ $F v\left(N_{w}\right)$ and therefore $x \in V_{a s s}\left(\delta_{2}^{\prime}\right)$. By hypothesis $x \in$ $V_{\text {ass }}\left(\delta^{\prime}\right) \cup V_{\text {stable }}\left(\delta_{1}^{\prime}\right)$. Then since $t$ is compatible with $\delta^{\prime}$ and $x \in V_{\text {loc }}(t)$, we have that $x \notin V_{a s s}\left(\delta^{\prime}\right)$ and therefore $x \in V_{\text {stable }}\left(\delta_{1}^{\prime}\right)$

4. We have that

$$
\begin{aligned}
& V_{l o c}\left(t^{\prime}\right) \cap V_{a s s}\left(\delta_{1}^{\prime}\right) \subseteq \\
& \quad\left(\text { since } V_{\text {loc }}\left(t^{\prime}\right)=V_{\text {loc }}(t)\right. \text { and since by hypothesis } \\
& \left.\quad A s s\left(\delta_{1}^{\prime}\right) \subseteq \text { Ass }\left(\delta^{\prime}\right) \uplus \operatorname{Stable}\left(\delta_{2}^{\prime}\right)\right) \\
& V_{l o c}(t) \cap\left(V_{a s s}\left(\delta^{\prime}\right) \cup V_{\text {Stable }}\left(\delta_{2}^{\prime}\right)\right) \subseteq \\
& \quad(\text { by }(10)) \\
& V_{l o c}(t) \cap\left(V_{\text {ass }}\left(\delta^{\prime}\right) \cup F v\left(G^{\prime}\right) \cup V_{l o c}\left(\delta_{2}^{\prime}\right)\right) \subseteq \\
& \quad\left(\text { since by hypothesis } V_{l o c}\left(\delta_{2}^{\prime}\right) \subseteq V_{l o c}\left(\delta^{\prime}\right)\right) \\
& V_{l o c}(t) \cap\left(V_{a s s}\left(\delta^{\prime}\right) \cup F v\left(G^{\prime}\right) \cup V_{l o c}\left(\delta^{\prime}\right)\right)= \\
& \quad\left(\text { since } t \text { is compatible with } \delta^{\prime} \text { and by definition of } V_{l o c}\right) \\
& \emptyset \quad
\end{aligned}
$$

Now assume that $\delta_{2}^{\prime} \in \mathcal{S}_{P}^{\prime}\left(G^{\prime}\right)$. Since by hypothesis $t$ is compatible with $\delta^{\prime}, V_{l o c}\left(\delta_{2}^{\prime}\right) \subseteq V_{l o c}\left(\delta^{\prime}\right)$ and $F v(G) \subseteq F v(t)$, we have that $F v\left(G^{\prime \prime}\right) \cap V_{l o c}\left(\delta_{2}^{\prime}\right)=\emptyset$. Then it is easy to check that $\delta_{2}=\delta_{2}^{\prime} \oplus G^{\prime \prime} \in \mathcal{S}_{P}^{\prime}(G)$ and then the thesis.

Finally, we prove that $\alpha\left(\delta_{1}\right) \| \alpha\left(\delta_{2}\right)$ is defined, i.e.

$$
\begin{aligned}
& \left(V_{l o c}\left(\alpha\left(\delta_{1}\right)\right) \cup F v(H)\right) \cap\left(V_{l o c}\left(\alpha\left(\delta_{2}\right)\right) \cup F v(G)\right) \subseteq \\
& F v(H) \cap F v(G) .
\end{aligned}
$$

Since $\alpha\left(\delta_{1}^{\prime}\right) \| \alpha\left(\delta_{2}^{\prime}\right)$ is defined, we have that

$$
\begin{aligned}
& \left(V_{l o c}\left(\alpha\left(\delta_{1}^{\prime}\right)\right) \cup F v\left(H^{\prime}, B\right)\right) \cap\left(V_{l o c}\left(\alpha\left(\delta_{2}^{\prime}\right)\right) \cup F v\left(G^{\prime}\right)\right) \subseteq \\
& F v\left(H^{\prime}, B\right) \cap F v\left(G^{\prime}\right) .
\end{aligned}
$$

By Lemma 1

$$
\begin{aligned}
& V_{l o c}\left(\alpha\left(\delta_{1}\right)\right)=V_{l o c}\left(\alpha\left(\delta_{1}^{\prime}\right)\right) \cup V_{l o c}\left(t^{\prime}\right) \text { and } \\
& V_{l o c}\left(\alpha\left(\delta_{2}\right)\right)=V_{l o c}\left(\alpha\left(\delta_{2}^{\prime}\right)\right) .
\end{aligned}
$$

Since $t$ is compatible with $\delta^{\prime}$ and by Lemma 1, we have that

$$
F v(t) \cap V_{l o c}\left(\alpha\left(\delta^{\prime}\right)\right)=\emptyset .
$$

Moreover, by hypothesis for $i=1,2 V_{l o c}\left(\alpha\left(\delta_{i}^{\prime}\right)\right) \subseteq V_{l o c}\left(\alpha\left(\delta^{\prime}\right)\right)$ and by definition of $t$, we have that $F v(H, G) \cup V_{l o c}\left(t^{\prime}\right) \subseteq F v(t)$. Then

$$
\begin{aligned}
& V_{l o c}\left(\alpha\left(\delta_{1}\right)\right) \cap\left(V_{l o c}\left(\alpha\left(\delta_{2}\right)\right) \cup F v(G)\right)= \\
& \left(V_{l o c}\left(\alpha\left(\delta_{1}^{\prime}\right)\right) \cup V_{l o c}\left(t^{\prime}\right)\right) \cap\left(V_{l o c}\left(\alpha\left(\delta_{2}^{\prime}\right)\right) \cup F v(G)\right)=\emptyset .
\end{aligned}
$$

Moreover by the previous observations

$$
\begin{aligned}
F v(H) \cap V_{l o c}\left(\alpha\left(\delta_{2}\right)\right) & =F v(H) \cap V_{l o c}\left(\alpha\left(\delta_{2}^{\prime}\right)\right) \\
& \subseteq F v(H) \cap V_{l o c}\left(\alpha\left(\delta^{\prime}\right)\right)=\emptyset
\end{aligned}
$$

and then the thesis holds. 
Lemma 6. (Lemma 2) Let $P$ be a program, $H$ and $G$ be two goals and assume that $\delta \in \mathcal{S}_{P}^{\prime}(H, G)$. Then there exists $\delta_{1} \in$ $\mathcal{S}_{P}^{\prime}(H)$ and $\delta_{2} \in \mathcal{S}_{P}^{\prime}(G)$, such that $\alpha(\delta) \in \eta\left(\alpha\left(\delta_{1}\right) \| \alpha\left(\delta_{2}\right)\right)$.

Proof We construct, by induction on the $l=$ length $(\delta)$ two sequences $\delta \uparrow_{(G . H)}=\left(\delta_{1}, \delta_{2}\right)$, where

1. for $i=1,2, V_{l o c}\left(\delta_{i}\right) \subseteq V_{l o c}(\delta)$ and $\operatorname{Inc}\left(\delta_{i}\right) \subseteq \operatorname{Inc}(\delta)$ (and therefore $C T \models$ instore $\left(\delta_{i}\right) \rightarrow$ instore $\left.(\delta)\right)$.

2. $\operatorname{Ass}\left(\delta_{1}\right) \subseteq \operatorname{Ass}(\delta) \uplus \operatorname{Stable}\left(\delta_{2}\right)$ and $\operatorname{Ass}\left(\delta_{2}\right) \subseteq \operatorname{Ass}(\delta) \uplus$ Stable $\left(\delta_{1}\right)$,

3. $\delta_{1} \in \mathcal{S}_{P}^{\prime}(H), \delta_{2} \in \mathcal{S}_{P}^{\prime}(G), \alpha\left(\delta_{1}\right) \| \alpha\left(\delta_{2}\right)$ is defined and

4. $\alpha(\delta) \in \eta\left(\alpha\left(\delta_{1}\right) \| \alpha\left(\delta_{2}\right)\right)$.

$(l=1)$ In this case $\delta=\langle(H, G), c, \emptyset,(H, G), c\rangle$. We define

$$
\delta \uparrow_{(H, G)}=(\langle H, c, \emptyset, H, c\rangle,\langle G, c, \emptyset, G, c\rangle)=\left(\delta_{1}, \delta_{2}\right),
$$

where $\delta_{1} \in \mathcal{S}_{P}^{\prime}(H)$ and $\delta_{2} \in \mathcal{S}_{P}^{\prime}(G)$. By definition for $i=1,2, V_{l o c}\left(\delta_{i}\right)=\emptyset$, Inc $\left(\delta_{i}\right)=\{c\}=\operatorname{Inc}(\delta)$ and $\operatorname{Ass}\left(\delta_{i}\right)=\emptyset$.

Moreover $\alpha\left(\delta_{1}\right)=\langle c, \emptyset, H, c\rangle$ and $\alpha\left(\delta_{2}\right)=\langle c, \emptyset, G, c\rangle$ and then $\alpha\left(\delta_{1}\right) \| \alpha\left(\delta_{2}\right)$ is defined. Now the proof is straightforward by definition of $\|$.

$(l>1)$ Assume that $\delta \in \mathcal{S}_{P}^{\prime}(H, G)$. By definition

$$
\delta=\left\langle(H, G), c_{1}, K_{1}, B_{2}, d_{1}\right\rangle \cdot \delta^{\prime},
$$

where $\delta^{\prime} \in \mathcal{S}_{P}^{\prime}\left(B_{2}\right)$ and $t=\left\langle(H, G), c_{1}, K_{1}, B_{2}, d_{1}\right\rangle$ is compatible with $\delta^{\prime}$. Recall that, by definition, the tuple $t$ represents a derivation step $s=\left\langle(H, G), c_{1}\right\rangle \longrightarrow^{K_{1}}\left\langle B_{2}, d_{1}\right\rangle$. Now we distinguish various cases according to the structure of the derivation step $s$.

- In the derivation step $s$, we use the Solve' rule. In this case, without loss of generality, we can assume that $H=$ $\left(c, H^{\prime}\right), s=\left\langle(H, G), c_{1}\right\rangle \longrightarrow^{\emptyset}\left\langle\left(H^{\prime}, G\right), d_{1}\right\rangle, C T \models$ $c_{1} \wedge c \leftrightarrow d_{1}, t=\left\langle(H, G), c_{1}, \emptyset,\left(H^{\prime}, G\right), d_{1}\right\rangle$ and $\delta^{\prime} \in$ $\mathcal{S}_{P}^{\prime}\left(H^{\prime}, G\right)$. Moreover $\alpha(\delta)=\left\langle c_{1}, \emptyset, W, d_{1}\right\rangle \cdot \alpha\left(\delta^{\prime}\right)$, where $W$ is the first stable set of $\alpha\left(\delta^{\prime}\right)$.

By inductive hypothesis there exist $\delta_{1}^{\prime} \in \mathcal{S}_{P}^{\prime}\left(H^{\prime}\right)$ and $\delta_{2} \in \mathcal{S}_{P}^{\prime}(G)$ such that $\delta^{\prime} \uparrow_{\left(H^{\prime}, G\right)}=\left(\delta_{1}^{\prime}, \delta_{2}\right), \alpha\left(\delta^{\prime}\right) \in$ $\eta\left(\alpha\left(\delta_{1}^{\prime}\right) \| \alpha\left(\delta_{2}\right)\right)$ and therefore there exists $\sigma^{\prime} \in \alpha\left(\delta_{1}^{\prime}\right) \|$ $\alpha\left(\delta_{2}\right)$ such that $\alpha\left(\delta^{\prime}\right) \in \eta\left(\sigma^{\prime}\right)$. Then, we define

$\delta \uparrow_{(H, G)}=\left(\delta_{1}, \delta_{2}\right)$ where $\delta_{1}=\left\langle H, c_{1}, \emptyset, H^{\prime}, d_{1}\right\rangle \cdot \delta_{1}^{\prime}$.

By definition $\left\langle H, c_{1}\right\rangle \longrightarrow^{\emptyset}\left\langle H^{\prime}, d_{1}\right\rangle$,

$t^{\prime}=\left\langle H, c_{1}, \emptyset, H^{\prime}, d_{1}\right\rangle$ represents a derivation step for $H, F v\left(d_{1}\right) \subseteq F v(H) \cup F v\left(c_{1}\right)$ and therefore $V_{l o c}\left(t^{\prime}\right)=$ $\emptyset$. Then the following holds.

1 . Let $i \in[1,2]$. By the inductive hypothesis, by construction and by the previous observation $V_{l o c}\left(\delta_{i}\right) \subseteq$ $V_{l o c}\left(\delta^{\prime}\right)=V_{l o c}(\delta)$ and $\operatorname{Inc}\left(\delta_{i}\right) \subseteq \operatorname{Inc}\left(\delta^{\prime}\right) \cup\left\{c_{1}\right\}=$ $\operatorname{Inc}(\delta)$.

2. By inductive hypothesis and by construction,

$$
\begin{aligned}
\operatorname{Ass}\left(\delta_{1}\right) & =\operatorname{Ass}\left(\delta_{1}^{\prime}\right) \subseteq \operatorname{Ass}\left(\delta^{\prime}\right) \uplus \operatorname{Stable}\left(\delta_{2}\right) \\
& =\operatorname{Ass}(\delta) \uplus \operatorname{Stable}\left(\delta_{2}\right) \text { and } \\
\operatorname{Ass}\left(\delta_{2}\right) & \subseteq \operatorname{Ass}\left(\delta^{\prime}\right) \uplus \operatorname{Stable}\left(\delta_{1}^{\prime}\right) \\
& =\operatorname{Ass}(\delta) \uplus \operatorname{Stable}\left(\delta_{1}\right) .
\end{aligned}
$$

3. By inductive hypothesis $\delta_{2} \in \mathcal{S}_{P}^{\prime}(G)$. Now, we prove that $\delta_{1} \in \mathcal{S}_{P}^{\prime}(H)$. By construction, we have only to prove that $t^{\prime}$ is compatible with $\delta_{1}^{\prime}$.
Since $V_{l o c}\left(t^{\prime}\right)=\emptyset$, we have that

$$
\begin{aligned}
& V_{\text {loc }}\left(t^{\prime}\right) \cap V_{\text {ass }}\left(\delta_{1}^{\prime}\right)=\emptyset \text { and } \\
& V_{\text {loc }}\left(t^{\prime}\right) \subseteq V_{\text {constr }}\left(t^{\prime} \cdot \delta_{1}^{\prime}\right) \cup V_{\text {stable }}\left(\delta_{1}^{\prime}\right) .
\end{aligned}
$$

Moreover, by inductive hypothesis, since $F v\left(t^{\prime}\right)=$ $F v(t)$ and since $t$ is compatible with $\delta^{\prime}$, we have that

$$
V_{l o c}\left(\delta_{1}^{\prime}\right) \cap F v\left(t^{\prime}\right) \subseteq V_{l o c}\left(\delta^{\prime}\right) \cap F v(t)=\emptyset .
$$

Then by the previous point 1 . and by definition, $t^{\prime}$ is compatible with $\delta_{1}^{\prime}$ and therefore $\delta_{1} \in \mathcal{S}_{P}^{\prime}(H)$.

Finally observe that by inductive hypothesis, $\alpha\left(\delta_{1}^{\prime}\right) \|$ $\alpha\left(\delta_{2}\right)$ is defined. Then since by construction $V_{l o c}\left(\delta_{1}\right)=$ $V_{l o c}\left(\delta_{1}^{\prime}\right)$ and by Lemma 1 , we have that

$$
V_{l o c}\left(\alpha\left(\delta_{1}\right)\right) \cap\left(V_{\text {loc }}\left(\alpha\left(\delta_{2}\right)\right) \cup F v(G)\right)=\emptyset .
$$

Moreover by inductive hypothesis and since $t$ is compatible with $\delta^{\prime}$, we have that $V_{l o c}\left(\delta_{2}\right) \cap F v(H) \subseteq$ $V_{l o c}\left(\delta^{\prime}\right) \cap F v(H)=\emptyset$ and therefore $\alpha\left(\delta_{1}\right) \| \alpha\left(\delta_{2}\right)$ is defined.

4. Now, observe that $\alpha\left(\delta_{1}\right)=\left\langle c_{1}, \emptyset, W_{1}, d_{1}\right\rangle \cdot \alpha\left(\delta_{1}^{\prime}\right)$, where $W_{1}$ is the first stable set of $\alpha\left(\delta_{1}^{\prime}\right)$. Then, since $\sigma^{\prime} \in \alpha\left(\delta_{1}^{\prime}\right) \| \alpha\left(\delta_{2}\right)$ we have that

$$
\left\langle c_{1}, \emptyset, W_{1} \uplus W_{2}, d_{1}\right\rangle \cdot \sigma^{\prime} \in \alpha\left(\delta_{1}\right) \| \alpha\left(\delta_{2}\right),
$$

where $W_{2}$ is the first stable set of $\alpha\left(\delta_{2}\right)$ and therefore $W^{\prime}=W_{1} \uplus W_{2}$ is the first stable set of $\sigma^{\prime}$. Then the thesis follows by observing that by the previous results and by definition of $\eta$,

$\alpha(\delta) \in \eta\left(\left\langle c_{1}, \emptyset, W^{\prime}, d_{1}\right\rangle \cdot \sigma^{\prime}\right) \subseteq \eta\left(\alpha\left(\delta_{1}\right) \| \alpha\left(\delta_{2}\right)\right)$.

- In the derivation step $s$, we use the Simplify' rule and let us to assume that in the derivation step $s$ atoms deriving from $H$ only are rewritten.

In this case, we can assume that $H=\left(H^{\prime}, H^{\prime \prime}\right), H^{\prime \prime} \neq$ $\emptyset, s=\left\langle(H, G), c_{1}\right\rangle \longrightarrow^{K_{1}}\left\langle\left(H^{\prime}, B, G\right), d_{1}\right\rangle, \delta^{\prime} \in$ $\mathcal{S}_{P}^{\prime}\left(H^{\prime}, B, G\right)$ and $t=\left\langle(H, G), c_{1}, K_{1},\left(H^{\prime}, B, G\right), d_{1}\right\rangle$. Moreover $\alpha(\delta)=\left\langle c_{1}, K_{1}, W, d_{1}\right\rangle \cdot \alpha\left(\delta^{\prime}\right)$, where $W$ is the first stable set of $\delta^{\prime}$ restricted to the atoms in $\left(H^{\prime}, G\right)$. By inductive hypothesis there exist $\delta_{1}^{\prime} \in \mathcal{S}_{P}^{\prime}\left(H^{\prime}, B\right)$ and $\delta_{2} \in \mathcal{S}_{P}^{\prime}(G)$ such that $\delta^{\prime} \uparrow_{\left(\left(H^{\prime}, B\right), G\right)}=\left(\delta_{1}^{\prime}, \delta_{2}\right)$, $\alpha\left(\delta^{\prime}\right) \in \eta\left(\alpha\left(\delta_{1}^{\prime}\right) \| \alpha\left(\delta_{2}\right)\right)$ and therefore there exists $\sigma^{\prime} \in \alpha\left(\delta_{1}^{\prime}\right) \| \alpha\left(\delta_{2}\right)$ such that $\alpha\left(\delta^{\prime}\right) \in \eta\left(\sigma^{\prime}\right)$ and $\alpha(\delta) \in$ $\eta\left(\left\langle c_{1}, K_{1}, W^{\prime}, d_{1}\right\rangle \cdot \sigma^{\prime}\right)$, where $W^{\prime}$ is the first stable set of $\sigma^{\prime}$ restricted to the atoms in $\left(H^{\prime}, G\right)$. Then, we define

$$
\begin{aligned}
& \delta \uparrow_{(H, G)}=\left(\delta_{1}, \delta_{2}\right) \text { where } \\
& \delta_{1}=\left\langle H, c_{1}, K_{1},\left(H^{\prime}, B\right), d_{1}\right\rangle \cdot \delta_{1}^{\prime} .
\end{aligned}
$$

By definition $\left\langle H, c_{1}\right\rangle \longrightarrow^{K_{1}}\left\langle\left(H^{\prime}, B\right), d_{1}\right\rangle$ and $t^{\prime}=$ $\left\langle H, c_{1}, K_{1},\left(H^{\prime}, B\right), d_{1}\right\rangle$ represents a derivation step for $H$ and $V_{l o c}\left(t^{\prime}\right)=V_{l o c}(t)$.

Now the following holds.

1. Let $i \in[1,2]$. By the inductive hypothesis, by construction and by the previous observation $V_{l o c}\left(\delta_{i}\right) \subseteq$ $V_{l o c}\left(\delta^{\prime}\right) \cup V_{l o c}(t)=V_{l o c}(\delta)$ and $\operatorname{Inc}\left(\delta_{i}\right) \subseteq \operatorname{Inc}\left(\delta^{\prime}\right) \cup$ $\left\{c_{1}\right\}=\operatorname{Inc}(\delta)$.

2. By inductive hypothesis and by construction,

$$
\begin{aligned}
& \operatorname{Ass}\left(\delta_{1}\right)=\operatorname{Ass}\left(\delta_{1}^{\prime}\right) \uplus\left\{K_{1}\right\} \subseteq \\
& \operatorname{Ass}\left(\delta^{\prime}\right) \uplus \operatorname{Stable}\left(\delta_{2}\right) \uplus\left\{K_{1}\right\}= \\
& \operatorname{Ass}(\delta) \uplus \operatorname{Stable}\left(\delta_{2}\right)
\end{aligned}
$$

and

$$
\operatorname{Ass}\left(\delta_{2}\right) \subseteq \operatorname{Ass}\left(\delta^{\prime}\right) \cup \operatorname{Stable}\left(\delta_{1}^{\prime}\right) \subseteq
$$$$
\operatorname{Ass}(\delta) \cup \operatorname{Stable}\left(\delta_{1}\right) \text {. }
$$ 
3. The proof follows by Lemma 5 .

4. By construction $\alpha\left(\delta_{1}\right)=\left\langle c_{1}, K_{1}, W_{1}, d_{1}\right\rangle \cdot \alpha\left(\delta_{1}^{\prime}\right)$, where $W_{1}$ is the first stable set of $\alpha\left(\delta_{1}^{\prime}\right)$ restricted to the atoms in $H^{\prime}$. Then $\left\langle c_{1}, K_{1}, W_{1} \uplus W_{2}, d_{1}\right\rangle$. $\sigma^{\prime} \in \alpha\left(\delta_{1}\right) \| \alpha\left(\delta_{2}\right)$, where $W_{2}$ is the first stable set of $\alpha\left(\delta_{2}\right)$ and therefore $W^{\prime}=W_{1} \uplus W_{2}$ is the first stable set of $\sigma^{\prime}$, restricted to the atoms in $\left(H^{\prime}, G\right)$. Then the thesis follows by observing that by the previous results and by definition of $\eta, \alpha(\delta) \in$ $\eta\left(\left\langle c_{1}, K_{1}, W^{\prime}, d_{1}\right\rangle \cdot \sigma^{\prime}\right) \subseteq \eta\left(\alpha\left(\delta_{1}\right) \| \alpha\left(\delta_{2}\right)\right)$.

- In the derivation step $s$, we use the Simplify' rule and let us to assume that in the derivation step $s$ atoms deriving both from $H$ and $G$ are rewritten.

In this case, we can assume that $H=\left(H^{\prime}, H^{\prime \prime}\right)$,

$G=\left(G^{\prime}, G^{\prime \prime}\right), H^{\prime \prime}, G^{\prime \prime} \neq \emptyset$,

$s=\left\langle(H, G), c_{1}\right\rangle \longrightarrow^{K_{1}}\left\langle\left(H^{\prime}, G^{\prime}, B\right), d_{1}\right\rangle$,

$\delta^{\prime} \in \mathcal{S}_{P}^{\prime}\left(H^{\prime}, G^{\prime}, B\right)$ and

$t=\left\langle(H, G), c_{1}, K_{1},\left(H^{\prime}, G^{\prime}, B\right), d_{1}\right\rangle$. Moreover $\alpha(\delta)=$ $\left\langle c_{1}, K_{1}, W, d_{1}\right\rangle \cdot \alpha\left(\delta^{\prime}\right)$, where $W$ is the first stable set of $\delta^{\prime}$ restricted to the atoms in $\left(H^{\prime}, G^{\prime}\right)$.

By inductive hypothesis there exist $\delta_{1}^{\prime} \in \mathcal{S}_{P}^{\prime}\left(H^{\prime}, B\right)$ and $\delta_{2}^{\prime} \in \mathcal{S}_{P}^{\prime}\left(G^{\prime}\right)$ such that $\delta^{\prime} \uparrow_{\left(\left(H^{\prime}, B\right), G^{\prime}\right)}=\left(\delta_{1}^{\prime}, \delta_{2}^{\prime}\right)$, $\alpha\left(\delta^{\prime}\right) \in \eta\left(\alpha\left(\delta_{1}^{\prime}\right) \| \alpha\left(\delta_{2}^{\prime}\right)\right)$ and therefore there exists $\sigma^{\prime} \in \alpha\left(\delta_{1}^{\prime}\right) \| \alpha\left(\delta_{2}^{\prime}\right)$ such that $\alpha\left(\delta^{\prime}\right) \in \eta\left(\sigma^{\prime}\right)$. Then, we define

$$
\begin{aligned}
& \delta \uparrow_{(H, G)}=\left(\delta_{1}, \delta_{2}\right) \text { where } \\
& \delta_{1}=\left\langle H, c_{1}, K_{1} \uplus\left\{G^{\prime \prime}\right\},\left(H^{\prime}, B\right), d_{1}\right\rangle \cdot \delta_{1}^{\prime} \text { and } \\
& \delta_{2}=\delta_{2}^{\prime} \uplus G^{\prime \prime} .
\end{aligned}
$$

By definition

$$
\left\langle H, c_{1}\right\rangle \longrightarrow^{K_{1} \uplus\left\{G^{\prime \prime}\right\}}\left\langle\left(H^{\prime}, B\right), d_{1}\right\rangle,
$$

$t^{\prime}=\left\langle H, c_{1}, K_{1} \uplus\left\{G^{\prime \prime}\right\},\left(H^{\prime}, B\right), d_{1}\right\rangle$ represents a derivation step for $H$ and $V_{l o c}\left(t^{\prime}\right)=V_{l o c}(t)$.

Now the following holds.

1. Let $i \in[1,2]$. The proof that $V_{l o c}\left(\delta_{i}\right) \subseteq V_{l o c}(\delta)$ and $\operatorname{Inc}\left(\delta_{i}\right) \subseteq \operatorname{Inc}(\delta)$ is analogous to the previous one and hence it is omitted.

2. By inductive hypothesis and by construction $A s s\left(\delta_{1}\right)=$ $\operatorname{Ass}\left(\delta_{1}^{\prime}\right) \uplus\left\{K_{1}\right\} \uplus\left\{G^{\prime \prime}\right\} \subseteq$ Ass $\left(\delta^{\prime}\right) \uplus \operatorname{Stable}\left(\delta_{2}^{\prime}\right) \uplus$ $\left\{K_{1}\right\} \uplus\left\{G^{\prime \prime}\right\}=\operatorname{Ass}(\delta) \uplus \operatorname{Stable}\left(\delta_{2}\right)$ and $\operatorname{Ass}\left(\delta_{2}\right)=$ $\operatorname{Ass}\left(\delta_{2}^{\prime}\right) \subseteq \operatorname{Ass}\left(\delta^{\prime}\right) \cup \operatorname{Stable}\left(\delta_{1}^{\prime}\right) \subseteq \operatorname{Ass}(\delta) \cup \operatorname{Stable}\left(\delta_{1}\right)$. 3. The proof follows by Lemma 5 .

4. By construction $\alpha\left(\delta_{1}\right)=\left\langle c_{1}, K_{1} \uplus\left\{G^{\prime \prime}\right\}, W_{1}, d_{1}\right\rangle$. $\alpha\left(\delta_{1}^{\prime}\right)$, where $W_{1}$ is the first stable set of $\alpha\left(\delta_{1}^{\prime}\right)$ restricted to the atoms in $H^{\prime}$. Then $\left\langle c_{1}, K_{1} \uplus\left\{G^{\prime \prime}\right\}, W_{1} \uplus W_{2} \uplus\right.$ $\left.\left\{G^{\prime \prime}\right\}, d_{1}\right\rangle \cdot \sigma^{\prime \prime} \in \alpha\left(\delta_{1}\right) \| \alpha\left(\delta_{2}\right)$, where $W_{2}$ is the first stable set of $\alpha\left(\delta_{2}^{\prime}\right)$ and $\sigma^{\prime \prime}$ is the sequence obtained from $\sigma^{\prime}$ by adding $\left\{G^{\prime \prime}\right\}$ to each stable set of $\sigma^{\prime}$. By construction $W^{\prime}=W_{1} \uplus W_{2}$ is the first stable set of $\sigma^{\prime}$, restricted to the atoms in $\left(H^{\prime}, G^{\prime}\right)$. Then the thesis follows by observing that by the previous results and by definition of $\eta, \alpha(\delta) \in \eta\left(\left\langle c_{1}, K_{1} \uplus\left\{G^{\prime \prime}\right\}, W^{\prime} \uplus\left\{G^{\prime \prime}\right\}, d_{1}\right\rangle \cdot \sigma^{\prime \prime}\right) \subseteq$ $\eta\left(\left\langle c_{1}, K_{1}, W^{\prime}, d_{1}\right\rangle \cdot \sigma^{\prime}\right) \subseteq \eta\left(\alpha\left(\delta_{1}\right) \| \alpha\left(\delta_{2}\right)\right)$.

LeMma 7. (Lemma 3) Let $P$ be a program, let $H$ and $G$ be two goals and assume that $\delta_{1} \in \mathcal{S}_{P}^{\prime}(H)$ and $\delta_{2} \in \mathcal{S}_{P}^{\prime}(G)$ are two sequences such that the following hold:

1. $\alpha\left(\delta_{1}\right) \| \alpha\left(\delta_{2}\right)$ is defined,
2. $\sigma=\left\langle c_{1}, K_{1}, W_{1}, d_{1}\right\rangle \cdots\left\langle c_{n}, \emptyset, W_{n}, c_{n}\right\rangle \in \eta\left(\alpha\left(\delta_{1}\right) \| \alpha\left(\delta_{2}\right)\right)$,

3. $\left(V_{l o c}\left(\alpha\left(\delta_{1}\right)\right) \cup V_{l o c}\left(\alpha\left(\delta_{2}\right)\right)\right) \cap V_{a s s}(\sigma)=\emptyset$,

4. for $i \in[1, n],\left(V_{l o c}\left(\alpha\left(\delta_{1}\right)\right) \cup V_{l o c}\left(\alpha\left(\delta_{2}\right)\right)\right) \cap F v\left(c_{i}\right) \subseteq$ $\bigcup_{j=1}^{i-1} F v\left(d_{j}\right) \cup \bigcup_{j=1}^{i} F v\left(W_{j}\right)$.

Then there exists $\delta \in \mathcal{S}_{P}^{\prime}(H, G)$ such that $V_{\text {loc }}(\delta) \subseteq V_{\text {loc }}\left(\delta_{1}\right) \cup$ $V_{l o c}\left(\delta_{2}\right)$ and $\sigma=\alpha(\delta)$.

Proof The proof is by induction on the $l=\operatorname{length}(\sigma)$.

$(l=1)$ In this case $\delta_{1}=\langle H, c, \emptyset, H, c\rangle, \delta_{2}=\langle G, c, \emptyset, G, c\rangle$, $\alpha\left(\delta_{1}\right)=\langle c, \emptyset, H, c\rangle, \alpha\left(\delta_{2}\right)=\langle c, \emptyset, G, c\rangle$, $\sigma=\langle c, \emptyset,(H, G), c\rangle$ and $\delta=\langle(H, G), c, \emptyset,(H, G), c\rangle$.

$(l>1)$ Without loss of generality, we can assume that

$$
\begin{aligned}
& \delta_{1}=t^{\prime} \cdot \delta_{1}^{\prime}, \delta_{2}=\left\langle G, e_{1}, J_{1}, G_{2}, f_{1}\right\rangle \cdot \delta_{2}^{\prime}, \\
& \sigma_{1}=\alpha\left(\delta_{1}\right)=\left\langle c_{1}, L_{1}, N_{1}, d_{1}\right\rangle \cdot \alpha\left(\delta_{1}^{\prime}\right) \text { and } \\
& \sigma_{2}=\alpha\left(\delta_{2}\right)=\left\langle e_{1}, J_{1}, M_{1}, f_{1}\right\rangle \cdot \sigma_{2}^{\prime},
\end{aligned}
$$

where $t^{\prime}=\left\langle H, c_{1}, L_{1}, H_{2}, d_{1}\right\rangle, \delta_{1}^{\prime} \in \mathcal{S}_{P}^{\prime}\left(H_{2}\right)$, $\sigma \in \eta\left(\left\langle c_{1}, L_{1}, N_{1} \uplus M_{1}, d_{1}\right\rangle \cdot \bar{\sigma}\right)$ and $\bar{\sigma} \in \alpha\left(\delta_{1}^{\prime}\right) \| \sigma_{2}$.

By definition of $\eta$, there exist the multisets of atoms $L^{\prime}, \bar{L}, L$ and the sequence $\sigma^{\prime}$ such that

$$
\sigma=\left\langle c_{1}, L_{1} \backslash L,\left(\left(N_{1} \uplus M_{1}\right) \backslash \bar{L}\right) \backslash L^{\prime}, d_{1}\right\rangle \cdot\left(\sigma^{\prime} \backslash L^{\prime}\right),
$$

where $\sigma^{\prime} \in \eta(\bar{\sigma}) \subseteq \eta\left(\alpha\left(\delta_{1}^{\prime}\right) \| \sigma_{2}\right), K_{1}=L_{1} \backslash L$ and $W_{1}=\left(\left(N_{1} \uplus M_{1}\right) \backslash \bar{L}\right) \backslash L^{\prime}$. Now the following holds

1. $\alpha\left(\delta_{1}^{\prime}\right) \| \alpha\left(\delta_{2}\right)$ is defined.

2. $\sigma^{\prime}=\left\langle c_{2}, K_{2}, W_{2} \uplus L^{\prime}, d_{2}\right\rangle \cdots\left\langle c_{n}, \emptyset, W_{n} \uplus L^{\prime}, c_{n}\right\rangle \in$ $\eta\left(\alpha\left(\delta_{1}^{\prime}\right) \| \alpha\left(\delta_{2}\right)\right)$.

3. By definition, by the hypothesis and by Lemma 1 , we have that

$$
\begin{aligned}
& \left(V_{l o c}\left(\alpha\left(\delta_{1}^{\prime}\right)\right) \cup V_{l o c}\left(\alpha\left(\delta_{2}\right)\right)\right) \cap V_{a s s}\left(\sigma^{\prime}\right) \subseteq \\
& \left(V_{l o c}\left(\alpha\left(\delta_{1}\right)\right) \cup V_{l o c}\left(\alpha\left(\delta_{2}\right)\right)\right) \cap V_{a s s}(\sigma)=\emptyset .
\end{aligned}
$$

4. For $i \in[2, n]$,

$$
\begin{aligned}
& \left(V_{l o c}\left(\alpha\left(\delta_{1}^{\prime}\right)\right) \cup V_{l o c}\left(\alpha\left(\delta_{2}\right)\right)\right) \cap F v\left(c_{i}\right) \subseteq \\
& \bigcup_{j=2}^{i-1} F v\left(d_{j}\right) \cup \bigcup_{j=2}^{i} F v\left(W_{j} \uplus L^{\prime}\right) .
\end{aligned}
$$

To prove this statement observe that by hypothesis and by Lemma 1 , for $i \in[2, n]$,

$$
\begin{aligned}
& \left(V_{l o c}\left(\alpha\left(\delta_{1}^{\prime}\right)\right) \cup V_{l o c}\left(\alpha\left(\delta_{2}\right)\right)\right) \cap F v\left(c_{i}\right) \subseteq \\
& \left(V_{l o c}\left(\alpha\left(\delta_{1}\right)\right) \cup V_{l o c}\left(\alpha\left(\delta_{2}\right)\right)\right) \cap F v\left(c_{i}\right) \subseteq \\
& \bigcup_{j=1}^{i-1} F v\left(d_{j}\right) \cup \bigcup_{j=1}^{i} F v\left(W_{j}\right) .
\end{aligned}
$$

Then to prove the thesis, we have only to prove that for $i \in[2, n]$, if $x \in\left(V_{l o c}\left(\alpha\left(\delta_{1}^{\prime}\right)\right) \cup V_{l o c}\left(\alpha\left(\delta_{2}\right)\right)\right) \cap F v\left(c_{i}\right)$ then $x \notin F v\left(d_{1}\right) \cup F v\left(W_{1}\right)$.

- Let $x \in V_{l o c}\left(\alpha\left(\delta_{1}^{\prime}\right)\right)$. Then since $t^{\prime}$ is compatible with $\delta_{1}^{\prime}$ and $W_{1} \subseteq H$, we have that $x \notin F v\left(t^{\prime}\right)$ and therefore $x \notin F v\left(d_{1}, W_{1}\right)$.

- Let $x \in V_{l o c}\left(\alpha\left(\delta_{2}\right)\right) \cap F v\left(c_{i}\right)$. By definition of $V_{l o c}$ and since $\alpha\left(\delta_{1}\right) \| \alpha\left(\delta_{2}\right)$ is defined, we have that

$$
x \notin F v(H) \cup F v(G) \cup V_{l o c}\left(\alpha\left(\delta_{1}\right)\right)
$$

and therefore, since $W_{1} \subseteq H$, we have that $x \notin$ $F v\left(W_{1}\right)$.

Now we prove by contradiction that $x \notin F v\left(d_{1}\right)$. Assume that $x \in F v\left(d_{1}\right)$. Since by previous result 
$x \not n W_{1}$, by point 4 . of the hypothesis $x \notin F v\left(c_{1}\right)$. Then, since by (11) $x \notin F v(H) \cup V_{l o c}\left(\alpha\left(\delta_{1}\right)\right)$, we have that $x \in F v\left(L_{1}\right)$. Moreover, since by hypothesis $V_{\text {loc }}\left(\alpha\left(\delta_{2}\right)\right) \cap V_{\text {ass }}(\sigma)=\emptyset$, we have that $x \notin F v\left(K_{1}\right)$. Then, since $K_{1}=L_{1} \backslash L$, we have that $x \in F v(L)$.

Now, observe that, by (11) and since $N_{1} \uplus M_{1} \subseteq$ $H \uplus G$, we have that $x \notin F v\left(L^{\prime}\right)$. Moreover by definition of $\eta, C T \models c_{1} \wedge L \leftrightarrow c_{1} \wedge L^{\prime}$. Then since $x \notin$ $F v\left(c_{1}\right)$, we have that $C T \models c_{1} \leftrightarrow$ false. Then, by definition of sequence for each $i \in[2, n]$, we have that $C T \models c_{i} \leftrightarrow$ false and $C T \models d_{i-1} \leftrightarrow$ false. Then by definition of $\mathcal{S}_{P}^{\prime}\left(H_{2}\right)$ and by definition of $\|$, we have that $\sigma_{1}=\left\langle c_{1}, \emptyset, H, c_{1}\right\rangle, \sigma_{2}=$ $\left\langle c_{1}, \emptyset, G, c_{1}\right\rangle$, length $\left(\sigma_{1}\right)=\operatorname{length}\left(\sigma_{2}\right)=1$ and this contradicts the hypothesis that $l=\operatorname{length}(\sigma)>$ 1.

By previous results and by inductive hypothesis, we have that there exists $\delta^{\prime} \in \mathcal{S}_{P}^{\prime}\left(H_{2}, G\right)$ such that $V_{l o c}\left(\delta^{\prime}\right) \subseteq V_{l o c}\left(\delta_{1}^{\prime}\right) \cup$ $V_{l o c}\left(\delta_{2}\right)$ and $\sigma^{\prime}=\alpha\left(\delta^{\prime}\right)$. Moreover by definition of $\eta, L^{\prime}$ is a multiset of atoms which are stable in $\delta^{\prime}$. Then $\tilde{\delta}=\delta^{\prime} \ominus L^{\prime} \in$ $\mathcal{S}_{P}^{\prime}(B)$, where the goal $B$ is obtained from the goal $\left(H_{2}, G\right)$ by deleting the atoms in $L^{\prime}$.

Now observe that since $t^{\prime}=\left\langle H, c_{1}, L_{1}, H_{2}, d_{1}\right\rangle$ represents a derivation step for $H$, we have that $t=\left\langle(H, G), c_{1}, K_{1}, B, d_{1}\right\rangle$ represents a derivation step for $(H, G)$. Let us denote by $\delta$ the sequence $t \cdot \tilde{\delta}$.

Then, to prove the thesis, we have to prove that $V_{l o c}(\delta) \subseteq$ $V_{l o c}\left(\delta_{1}\right) \cup V_{l o c}\left(\delta_{2}\right), t$ is compatible with $\tilde{\delta}$ (and therefore $\delta \in$ $\left.\mathcal{S}_{P}^{\prime}(H, G)\right)$ and $\sigma=\alpha(\delta)$.

$\left(V_{l o c}(\delta) \subseteq V_{l o c}\left(\delta_{1}\right) \cup V_{l o c}\left(\delta_{2}\right)\right)$. By construction

$\begin{array}{lll}V_{l o c}(\delta) & =\text { by construction } \\ V_{l o c}(t) \cup V_{l o c}(\tilde{\delta}) & =\text { by construction } \\ V_{l o c}\left(t^{\prime}\right) \cup V_{l o c}\left(\delta^{\prime}\right) & \subseteq & \text { by inductive hypothesis } \\ V_{l o c}\left(t^{\prime}\right) \cup V_{l o c}\left(\delta_{1}^{\prime}\right) \cup V_{l o c}\left(\delta_{2}\right) & =\text { by construction } \\ V_{l o c}\left(\delta_{1}\right) \cup V_{l o c}\left(\delta_{2}\right) & \end{array}$

and then the thesis.

( $t$ is compatible with $\tilde{\delta}$ ). The following holds.

1. $C T \models$ instore $(\tilde{\delta}) \rightarrow d_{1}$. The proof is straightforward, since by construction either instore $(\tilde{\delta})=$ instore $\left(\delta_{1}^{\prime}\right)$ or instore $(\tilde{\delta})=$ instore $\left(\delta_{2}\right)$.

2. $V_{l o c}(\tilde{\delta}) \cap F v(t)=\emptyset$. By construction and by inductive hypothesis

$$
\begin{aligned}
& V_{l o c}(t)=V_{l o c}\left(t^{\prime}\right), F v(t) \subseteq F v\left(t^{\prime}\right) \cup F v(G) \text { and } \\
& V_{l o c}(\tilde{\delta}) \subseteq V_{l o c}\left(\delta_{1}^{\prime}\right) \cup V_{l o c}\left(\delta_{2}\right) .
\end{aligned}
$$

Since $t^{\prime}$ is compatible with $\delta_{1}^{\prime}$, we have that and $\alpha\left(\delta_{1}^{\prime}\right) \| \alpha\left(\delta_{2}\right)$

$$
V_{l o c}\left(\delta_{1}^{\prime}\right) \cap\left(F v\left(t^{\prime}\right) \cup F v(G)\right)=\emptyset .
$$

By points 3. and 4. of the hypothesis $F v\left(K_{1}, c_{1}\right) \cap$ $V_{l o c}\left(\delta_{2}\right)=\emptyset$ and by points 1 . of the hypothesis we have that $\alpha\left(\delta_{1}\right) \| \alpha\left(\delta_{2}\right)$ is defined and therefore $\left(F v(H) \cup V_{l o c}\left(t^{\prime}\right)\right) \cap V_{l o c}\left(\delta_{2}\right)=\emptyset$. Then by definition and by (12)

$$
\begin{aligned}
& F v(t) \cap V_{l o c}\left(\delta_{2}\right)= \\
& \left(F v\left(c_{1}, H, K_{1}\right) \cup V_{l o c}\left(t^{\prime}\right)\right) \cap V_{l o c}\left(\delta_{2}\right)=\emptyset .
\end{aligned}
$$

Then

$V_{l o c}(\tilde{\delta}) \cap F v(t) \subseteq$ (by the last statement in (12))

$\left(V_{l o c}\left(\delta_{1}^{\prime}\right) \cup V_{l o c}\left(\delta_{2}\right)\right) \cap F v(t) \subseteq$ (by the second statement in (12) and by (13))

$V_{l o c}\left(\delta_{2}\right) \cap F v(t)=$ (by definition, by the first statement in (12) $\emptyset$. and by (14))

3. for $i \in[2, n], V_{l o c}(t) \cap F v\left(c_{i}\right) \subseteq \bigcup_{j=1}^{i-1} F v\left(d_{j}\right) \cup$ $V_{\text {stable }}(\tilde{\delta})$. The proof is immediate by construction and by point 4 . of hypothesis.

4. $V_{\text {loc }}(t) \cap V_{\text {ass }}(\tilde{\delta})=\emptyset$. The proof is immediate by point 3. of the hypothesis.

$\left(\sigma=\alpha(\delta)\right.$ ). By inductive hypothesis $\sigma^{\prime}=\alpha\left(\delta^{\prime}\right)$ and then by construction $\sigma^{\prime} \backslash L^{\prime}=\alpha(\tilde{\delta})$. Then

$$
\begin{aligned}
\sigma & =\left\langle c_{1}, K_{1}, W_{1}, d_{1}\right\rangle \cdot\left(\sigma^{\prime} \backslash L^{\prime}\right) \\
& =\left\langle c_{1}, K_{1}, W_{1}, d_{1}\right\rangle \cdot \alpha(\tilde{\delta}) \\
& =\alpha(\delta)
\end{aligned}
$$

where the last equality follows by observing that $\delta=t \cdot \tilde{\delta}$, where

$$
t=\left\langle(H, G), c_{1}, K_{1}, B, d_{1}\right\rangle
$$

and $W_{1}$ is the multiset of all the atoms in $(H, G)$, which are stable in $\delta$. 